\title{
Perceived Risk of Sexual Harassment and Protective Behaviours among Zambian Soldiers in Selected Military Camps
}

\author{
John Mwitumwa Matakala (Ph.D.)*, Prof. Mubiana Macwan'gi, Dr. Anne Namakando- Phiri \\ (Col RtD). \\ Zambia
}

*Corresponding Author: John Mwitumwa Matakala, Zambia

\begin{abstract}
The aim of the thesis was to explore the link between Perceived Risk of being sexually harassed and protective behaviours adopted by soldiers in selected military camps. In order to understand the lived experiences and interpretations related to the topic, the research made use of a qualitative inquiry that was grounded in the Hermeneutic Phenomenology Design. Purposive Sampling was based on the selection criteria of the Sexual Harassment Experience Questionnaire (SHEQ) inorder to come up with the desired sample size of 95 (74 victims and 21 non-victims). The Self-Report Response strategy was used at participant individual level so as to ensure protection based on anonymity and confidentiality. Data were collected using the Semi-Structured Interview Guide.

The findings were categorized in line with the objectives of the study which where to:(a) explore perceived risk perceptions of being sexually harassed (b) determine the risk factors associated with sexual harassment.(c) assess the protective behaviors against the risk factors of sexual harassment.(d) examine how perceived risk of being sexually harassed motivates protective behaviors.

The findings on perceived risk situation of being sexually harassed revealed a high magnitude for both unwelcome verbal and non-verbal actions without any punishment. Only ranks between private and corporal continued being affected. The ranks above sergeant only experienced these actions between the ranks of private and corporal. This situation gave rise to a high perceived risk of being sexually harassed among the current affected category victim and non-victim soldiers.

The findings on the risk factors associated with sexual harassment were measured as (i) perpetrator (ii) individual weakness and (iii) military characteristics respectively. This was also done from the perspective of both victims and non-victim soldiers. Male/Female bully and discriminatory was among the recorded perpetrator characteristics. Furthermore, non-reporting of the perpetrators was recorded as an individual victim weakness which was not the case for the non-victims. Lack of written sexual harassment mitigation measures was recorded as a military characteristic that was also a risk factor for the prevalence of sexual harassment.
\end{abstract}

The findings on protective behaviors against risk factors of sexual harassment deviated from existing theory. It was established victims never adopted protective behaviors fearing revenge, but non-victims protected themselves as they feared getting affected health-wise. In both cases the new explanation of phenomenon was a motivation against high perceived or awareness of risk factors of sexual harassment. This was taken from an initial measurement of (low, medium and high) levels risk. It was calculated using a $3 * 3$ risk analysis matrix of which the risk had two components possibility and severity. This led to (Matakala 2021)'s theory and others.

The findings from the non-victim on how the high perceived risk of being sexually harassed motivated protective behaviors revealed three protective initiatives. These were escape and evasion, stick and avoidance of lone movement protective initiatives. The escape and evasion was based on the principle of being able to see the known perpetrator first so that it is easy to avoid them. The avoidance of lone movement principle was meant not to come in the presence of the known perpetrator while alone. Lastly, the stick principle was made to ensure movement to the known perpetrator was done in a group of four making it difficult for harassment to take place.

The research originality, which is a major significant contribution to the knowledge base, concluded the study, with a scholarly demonstration through the use of the non-victim protective initiatives against sexual harassment, which were later subjected to validation by selected victim soldiers, who initially lacked the said initiatives, but had the awareness of risk factors.

While the findings of soldiers could also be extrapolated to officers, it is important future studies consider the category.

Keywords: Perceived Risk, Sexual Harassment, Soldiers and Military camps. 


\section{INTRODUCTION}

\subsection{Background and Context Of The Research}

\section{Context of the Topic (Sexual Harassment and Zambian Soldiers)}

Sexual harassment is currently a growing global gender concern with a gendered dimension that embraces the sex of both males and females. It includes physical, verbal and non-verbal unwelcome actions that affect a number of organisations including the military in Africa (UNSC, 2008). According to UNSC (2008), 50\% female and 30\% male soldiers are sexually harassed in Southern Africa. Conformity, obedience, and the different hierarchical gender power relations between those in the lower ranks and higher ranks are a major factor that has contributed to the existence of sexual harassment in the military organizations (UNSC, 2008). The working morale and execution of military duties by the lower ranks is lowered because of being sexually harassed by the higher ranks. Sexual harassment is also a public health issue which is linked to other severe long term health problems that could put one at the risk of high blood pressure, anxiety, depression and insomnia (WHO, 2014). This because sexual harassment involves unwelcome physical, verbal and non-verbal actions on a victim.

Zambia is one of the Southern African countries that have tried to deal with the problem of sexual harassment in the country as a whole including military organizations. This has been done by enacting the section 137(A) Statutory Instruments No. 15 of 2005. This (SI) highlights the types of sexual harassment and the punishment against perpetrators of sexual harassment. These include unwelcome verbal, non-verbal and physical sexual harassment with sentences ranging from 3 to 15 years for perpetrators (GRZ, 2005). The State has therefore, criminalized sexual harassment in Zambia which also includes the military. An extract from section 137, Sub-Section 1, 2 and 3 of the penal code with relevant laws on workplace sex harassment is highlighted below:

\section{$137 A$}

(1) Any person who practices sexual harassment in a work place, institution of learning or elsewhere on a child or an adult commits felony and is liable. Upon conviction, to an imprisonment for a term of not less than (03) three years and not exceeding (15) fifteen years imprisonment.

(2) A child who commits offence under subsection (1) is liable to community service or counseling as the court may determine in the best interests of the child.

(3) In this section, sexual harassment means (a) a seductive sexual advance being unsolicited sexual comment, physical contact or other gesture of sexual nature which one finds objectionable or offensive or which cause discomfort in one's studies or job and interferes with academic performance or conducive working or study environment; (b) sexual bribery in the form of soliciting or attempting to solicit sexual activity by promise of reward; (c) sexual threat or coercion which includes procuring or attempting to procure sexual activity by threat of violence or victimization; or (d) sexual imposition using forceful behavior or assault in an attempt to gain physical sexual contact.

The implementation of the statutory instrument act No. 15 of 2005 section 137 of the penal code chapter 87 of the laws of was one of the legislation measures that was considered by the Zambian government because of the admonition by the Convention on the Elimination of All Forms of Discrimination Against Women (CEDAW). This is because all the signatories of which Zambia is a part agreed to come up with pieces of legislation that would avoid discrimination of women in line with social and cultural issues that bring about sexual harassment and gender based violence (GRZ and GIDD, 2007).

The Zambian government through the Ministry of Gender has also come up with the National Gender Policy which has also admonished the headquarters of government ministries such as the Ministry of Defence to ensure that vulnerable groups such as women and girls are protected from sexual harassment (GRZ, 2014). Section 6.1.3.5, In particular admonished the Ministry of Defence and its three security wings which are Zambia Air force (ZAF), Zambia National Service (ZNS) and Zambia Army to ensure that vulnerable and marginalised groups such as women and girls within the security wings and outside are protected from sexual harassment. This should be done in the most effective 
and efficient way when preserving and protecting as well as defending the sovereignty of the nation in both peace time and active service. The ministry of defence alongside its three security wings have since abided, Zambia Army inclusive.

The National Gender Policy (NGP) was created as an awareness tool concerning the affirmative response towards discriminatory activities such as sexual harassment. The government succeeded in implementing the National Gender Policy (NGP) through the collaboration of civil society organisations such as Non-Governmental Gender Organisation Coordinating Council (NGOCC); Women and Law in Southern Africa (WLSA); Women in Law and Development in Africa (WILDAF). By working together the government was trying to disseminate information on the implementation of (CEDAW) through the National Gender Policy (GRZ and GIDD, 2007).

The Zambian government through the Ministry of Gender has also proposed the enactment of Statutory Instrument (SI) number 22 of 2015 which advocates for Gender Equality and Equity in trying to deal with the problem of sexual harassment in the country as a whole including military organizations. This has been done by enacting the section 39 [prohibition of sexual harassment] and section 40 [policy and procedures of sexual harassment] (GRZ, 2015). The Ministry of Gender was responding to the Southern African Development Committee (SADC) protocol on Gender Development that was signed in Johannesburg, South Africa in 2008. The government therefore, admonished various headquarters of government ministries such as the Ministry of Defence alongside its three security wings Zambia Army inclusive to ensure they not only prohibit sexual harassment but also come up with procedures that allow victims to complaint without intimidation.

The Zambian government through the Ministry of Gender has also proposed that the section 39 [prohibition of sexual harassment] and section 40 [policy and procedures of sexual harassment] from Statutory Instrument (SI) number 22 of 2015, be reemphasised in the Seventh National Development Plan (SNDP=2017-2021). The Seventh National Development Plan (SNDP) in trying to transition from a low to middle income status through the vision 2030 has since called for various headquarters of ministries to introduce Gender Desks for sensitisation of sexual harassment (GRZ, 2017). A cooperating partner in ensuring the successful completion of the national development plan was the United Nations Fund for Population Agency (UNFPA) a voluntary funded international organisation that fights for both equal rights for the marginalised groups such as women and girls

According to UNFPA (2018) through the Zambian Annual Report, key milestones regarding the establishment of gender desks in the headquarters of government ministries and departments including the Ministry of Defence were recorded. Individual military organisations in Zambia such as the Zambia Army have also established gender desks at their headquarters. The main function of the gender desks is to sensitise workers on gender issues including fighting against sexual harassment. The gender desk roles have also been extended to troops that go out of the country for peace keeping missions.

The Gender Desks was a strategy that was meant to create awareness about human rights socialisations that would avoid sexual harassment in work places. The project was to be kick started by government in the first quarter of 2008 through the process of a Gender and Development Communication Strategy in Zambia.

Sexual harassment in the military, in Zambia presents itself in form of unwelcome verbal and nonverbal actions (Matakala e'tal, 2018). This study intends to understand the protective behaviours that soldiers who perceive risk of being sexually harassed adapt.

\section{Motivation for studying protective behaviours that Zambian soldiers who perceive risk of being sexually harassed adapt.}

The motivation for studying protective behaviours that soldiers who perceive risk of being sexually harassed adapt, is because the identified research gap is an under explored area in the Zambian context. Research studies have been conducted on sexual harassment in other militaries such as Gallagher (2008), Turchik and Susan (2010), Tangri and Hunson (2002) Valerie and Cynthia (2016), and Bell e'tal (2018), including the Zambian military, Matakala e'tal (2018).

Despite, these many studies, few studies have examined the concept of perceived risk and sexual harassment. One of the reviewed studies by Fontalvo et al (2019) explored the factors that influence 
female victim's perceived risk of sexual harassment in a bus rapid transit system in Barranquilla town of Colombia. The study didn't look at protective behaviours and this was a non-military community. Bell e'tal (2018), also used the concept of perceived risk on the United States Army, but only considered a sub-population of female victims hence the motivation to also include non-victim in the Zambian context.

Perceived Risk is an important concept in understanding of the different adopted protective behaviours for sexual harassment. Key for this study is that people with Low perceived risk; arenot likely to come up with protective behaviors. While those with High perceived risk; are likely to come up with protective behaviors (Ferrer e'tal, 2016). Therefore, the findings may also contribute to the prevention of sexual harassment among Zambian soldiers (Ferrer e'tal, 2016 and Fontalvo e'tal).

\subsection{Statement of the Problem}

The Zambian military has domesticated the penal code law, in particular section 137 A (SI) laws of 2005, Statutory Instrument (SI) number 15 of 2005, which regards sexual harassment as a crime. In addition the military has established the gender desks, with the overall aim of sensitizing the soldiers about sexual harassment. Further, research has also been done by Matakala and others in 2018 as well as in 2015 accompanied with solutions that could reduce sexual harassment in the Zambian military context. Despite, these efforts 50\% female and 30\% male soldiers are sexually harassed in Southern Africa, including Zambia (UNSC 2008 in Matakala e'tal, 2018). Sexual harassment in the military, in Zambia presents itself in form of unwelcome verbal and non- verbal actions (Matakala e'tal, 2018).

This shows that soldiers are still exposed to sexual harassment. This therefore, raises questions about the current situation on the perceived risk perceptions of being sexually harassed. This further raises questions regarding awareness of risk factors of sexual harassment. This situation also raises questions on whether or not soldiers who perceive the risk of sexual harassment are aware of and/or adopt protective behaviours. The answers to the raised questions lie in the exploration of lived experiences based on the participant perspectives of the present study.

Although, past studies have been conducted on sexual harassment, only few studies have used the concept of perceived risk. Scientific Research/Theory shows that Perceived Risk which is measured against three awareness levels (no, low and high) is an important construct because it helps in determining whether or not victims of a health/social threat of (being sexually harassed) protect themselves, so that mitigation measures can be proposed (Ferrer e'tal, 2016 and Fontalvo e'tal). It is therefore, important to explore and understand, link between Perceived Risk of being sexually harassed and protective behaviours adopted by soldiers if mitigation measures are to be proposed for the military camps, as it is an under explored area.

\subsection{Purpose of the Study}

This Phenomenological study therefore, attempts to make a contribution to the knowledge base by showing the link between Perceived Risk of being sexually harassed and protective behaviours adopted by soldiers.

\subsection{Specific Objectives}

(1)To explore perceived risk perceptions of being sexually harassed among Zambian soldiers in selected military camps.

(2) To determine the risk factors associated with sexual harassment among Zambian soldiers in selected military camps.

(3) To assess the protective behaviors against the risk factors of sexual harassment among Zambian soldiers in selected military camps.

(4) To examine how perceived risk of being sexually harassed motivates protective behaviors among Zambian soldiers in selected military camps.

\subsection{Research Questions}

(1) How is the situation of perceived risk perceptions of being sexually harassed among Zambian soldiers in selected military camps? 
(2) What risk factors are associated with sexual harassment among Zambian soldiers in selected military camps?

(3) What protective behaviors do Zambian soldiers adopt against the risk factors of being sexually harassed in selected military camps?

(4) How do perceived risks of being sexually harassed motivate protective behaviors among Zambian soldiers in selected military camps?

\subsection{Significance of the Study}

The study is important because it gives an insight into the different protective behaviors that Zambian Soldiers who perceive risk of sexual harassment in selected military camps utilize. It also shows the awareness levels of risk factors associated with sexual harassment. It is also hoped that the study will act as a stepping stone for further research.

\subsection{Ethical Consideration}

Ethical clearance was obtained from the ethics committee of the University of Zambia. Having initially conducted a similar study in 2015 , permission was once again sought from the military authorities' inorder to access entry. Permission was also sought from the actual participants. The 'Sexual Harassment Experience Questionnaire' (SHEQ) used for identification and accurate description of participants, whose answers required an affirmative response regarding the definition of study concepts such as perceived risk and sexual harassment were assured of confidentiality because it was done at individual level using the Self-Report Response Strategy. Having been identified through the 'Sexual Harassment Experience Questionnaire' (SHEQ) was at individual level soldiers were also assured of anonymity, confidentiality and being untraceable before and after the study because the actual interviews were also done at individual level using Self-Report Response Strategy. The assurance and trust in the researcher was further strengthened because they were told that no actual names would be recorded but pseudonyms. All efforts were further taken to ensure that the rights of participants as per research ethics are protected and respected. Participants are assured that they are free to ask for clarification at any point of the exercise and to inform the researcher if they feel uncomfortable about any procedure in the research. It is against this background that the few female participants that were not comfortable to reveal certain information based on sex differentiation with the researcher were availed with a trained female research assistant. This was based on their verbal consent. They were also told that they were free to withdraw from the study at any time that is if a participant does not want to respond to a question. He/She was free to say so or to leave it and go to the next question, interviews were held at a convenient place and time of their choice.This made it easy for the soldiers to easily open up. This in turn led to an in-depth understanding of the phenomenon because trust was enhanced. Soldiers were further told that participation is voluntary and as such they have the right to pullout when the feel uncomfortable. Verbal or Written consent was then obtained.

\subsection{Theoretical Frameworks}

This study was guided by three theories identified through the use of the narrative literature review based methodology as shown hereunder;

\subsubsection{Tripartite Perceived Risk Model}

It gives an insight into the perceived risks of a health threat and peoples subjective judgements about whether/whether or not they should develop protective behaviours based on possibility and severity knowledge.

Key for this study is that people with Low perceived risk; are not likely to come up with protective behaviors. While those with High perceived risk; are likely to come up with protective behaviors. (Ferrer e'tal, 2016).

Perceived Risk model is relevant to sexual harassment as it falls within the social and health definition for which this model was developed for. Sexual harassment is a public health issue which is linked to other severe long term health problems that could put one at the risk of high blood pressure, anxiety, depression and insomnia that may arise from physical, verbal and unwelcome actions (WHO, 2014). 
The justification for the use of this validated descriptive theory or model lies in its ability of describing and classifying the concept of perceived risk in terms of the corresponding subjective individual protective behavioural characteristics by patients against a health threat as an explanation. Descriptive model/theories are important in areas were nothing or very little is known such as the understanding of Non-victim/ Victim protective behaviours in relation to the perceived risk of being sexually harassed among Zambian soldiers (Carey, 2012).

As per research principles the present research can replicate the model by utilising the questions that it used for drawing its conclusions with a view to confirm, reject or reform the model (Moody, 1990).

The adapted risk model is further contextualized with gender power relations theory so as to understand how conformity, obedience, and hierarchical power relations between those in the lower ranks and higher ranks may contribute to sexual harassment. The relationship is the determination of how they protect themselves when they perceive the risk.

The adapted risk model is further contextualized with the token theory so as to understand whether the minority situation for females can lead to sexual harassment. The relationship is the determination of how they protect themselves when they perceive the risk.

\subsubsection{Theory of gender power relations}

Those who hold power and authority from either sex are likely to sexually harass the juniors due to differential hierarchical gender power relations existing within the different rank structures. The observations are based on large military camps headed by field rank because they are potential sexual harassment areas (Foucault, 1975).

\subsubsection{Theory of tokenism}

The theory of tokenism refers to the discrimination and marginalization of the members of a group in a minority position. This theory proposes that members of any social group ranging from political areas, military organizations, schools and other groups up to less than $15 \%$ are discriminated. This theory further states that if these minority groups are not represented by the governing of the affairs of that organization their complaints will be dying a natural death. The theory of tokenism was developed using evidence from women's experiences of sexual harassment and marginality in male occupations (Kamir, 1998).

In this study attention is drawn to the theory of tokenism as a guide to the present inquiry. This is because the main thrust of the theory is that groups in the minority are more likely to be marginalized by a group in the majority. The justification on the use of the theory as a guide is because the study also will look at female soldiers who are in the minority. Furthermore, the justification is that the theory has been used and it worked in a study conducted by (Zandonda, 2010) in Ndola under similar conditions. The theory also worked for other studies by (Matakala;2015 and Matakala 2018).The present inquiry therefore uses the aforementioned theory because of the principle of replication which advocate that an inquiry can use a theory used by another study in similar conditions hence being transmittable to the present inquiry Bless and Achola (1988). The findings of this study being similar can be compared to the theory in terms of whether or not they agree with the theory.

\subsubsection{Conceptual Framework of the study}

A conceptual framework in a qualitative research is one that involves the synthesis of concepts/constructs from existing literature in relation to the research problem of the thesis inorder to come up with research objectives/ questions that best explain the natural progression of the phenomenon of sexual harassment that is being studied in relation to perceived risk concept.

This framework is therefore, based on the synthesis of existing literature by the researcher, from the perceived risk model and other sexual harassment studies. Taken together these act as a conceptual road map for the present inquiry.

\section{$>$ Study Conceptual Framework}

The study assumes that, measurement of Military organizations, Sexist/Perpetrators and Individual Potential Risk Factors as an (Independent Variable or Construct) may be responsible for Sexual Harassment whose measurements includes physical, verbal and non-verbal unwelcome actions as the 
(Dependent Variable or Construct). The study further assumes that measurement of Low Risk, Medium Risk and High Risk attributes for Perceived Risk perceptions of soldiers as a (Moderating Independent Variable or Construct) will help in strengthening the initial relationship between the independent and dependent variable above. Lastly the study assumes that Practically-Validated Protective Behaviors for soldiers may be the (Resultant Research Output).

\section{$>$ Measurements or Observations of the major constructs in the relationships of the Study Conceptual Framework.}

The conceptual framework constructs are based on validated-reliable theoretical evidence. These are the Tririsk model for perceived risk (Ferrer et al, 2016), Tripartite model for sexual harassment (Fitzgerald et al, 1995) and the Four Factor Model which identifies Potential Risk Factors associated with sexual harassment (Elizabeth O'Hare and William Donohue, 1998).

Measurements or Observations for the major concepts or constructs will be based the extraction of questions that have been validated by theory as confirmation. The concepts include perceived risk, potential risk factors and sexual harassment.

a) Perceived Risk, Measurements or Observations in the present study are based on the three validated categories which include; Low Perceived Risk, Medium Perceived Risk and High Perceived Risk. These will form part of the thematic questions as observations regarding the concept and how it relates with the topic of interest (sexual harassment).

The source for replication of the measurements or observations is based on the validated works of the Tririsk model for perceived risk (Ferrer et al, 2016).

b) Potential Risk Factor Measurements or Observations in the present study are based on the three validated categories which include the Organizations (Military), Sexist/Perpetrators (Senior Soldiers) and Individual (The Harassed). These will form part of the thematic questions as observations regarding the concept and how it relates with the topic of interest (sexual harassment). The source for replication of the measurements or observations is based on the validated works of the Tririsk model for perceived risk (Elizabeth O'Hare and William Donohue, 1998).

c) Sexual Harassment Measurement or Observations in the present study are based on three validated categories which include physical, verbal and non-verbal unwelcome actions on the (The harassed) all of which were validated through the confirmatory analysis taking into account gender harassment, sexual coercion and unwanted sexual attention. These will form part of the thematic questions as observations in relation to other concepts of perceived risk and risk factors. The source for replication of the measurements or observations is based on the validated works of the tripartite model for sexual harassment (Fitzgerald et al, 1995).

The researcher identified constructs that formed relationships explaining the natural progression of this study based the identified research problem/research questions in line with the qualitative conceptual framework principles (Miles and Huberman, 1999 \& Guba and Lincoln, 1994). Furthermore, this explanatory conceptual framework which is the researcher's perception has been demonstrated in word or narrative form. This is according to the research principles hence the justification (Holloway and Wheeler, 1996).

\section{RESEARCH METHODOLOGY}

\subsection{Research Paradigm}

This study uses phenomenology and ethno methodology as paradigms to locate the study. Research questions number one and two will be studied using phenomenology.

\section{Phenomenology Paradigm.}

Phenomenology is interested in meanings of the things that people do. The lived experiences simply mean everyday encounters that people go through. This has been clearly described by the two variants Husserlian and Haiddegerian. The Husserlian being ascribed to Edmund Hursell while Haiddegerian 
is ascribed to Martin Heiddger.Phenomenology was chosen for this study because the research desires to appreciate, how the concept of Perceived Risk of sexual harassment among Zambian soldiers is associated with protective behaviours in selected military camps with respect to two sub-population groups-Victims and Non-Victims in relation to their experiences.

This fits well with the assumptions of phenomenology according to Moustakas (1994). Phenomenology also helps in describing phenomena from the participants' lay verbal accounts

(1) How is the situation of perceived risk of being sexually harassed among Zambian soldiers in selected military camps?

(2) What risk factors are associated with sexual harassment among Zambian soldiers in selected military camps?

\section{Ethno-Methodology Paradigm.}

Whereas Ethnomethodology are only interested in what makes sense for them and the methods they use to protect themselves (Garfinkel, 1967). It was popularized by Harold Garfinkel. It will be combined the Tripartite Model of Risk Perception as it has two types of risk perceptions which are low and high that will be used inorder to understand how they are associated with protective behaviours which in this case are against sexual harassment. Key for this work is that soldiers with a low perceived risk perception are less likely to take up any protective behaviour against sexual harassment. While those with a high perceived risk perception are more likely to take up any protective behaviour against sexual harassment. This is the main focus of this study based on the principles of the Tripartite Model of Risk Perception (Ferrer E'tal, 2016). See (2.2).

These protective behavioral strategies are clearly elaborated under the literature review section but targeted for the third question shown hereunder:

(3) What protective behaviors do Zambian soldiers adopt against the risk factors of being sexually harassed in selected military camps?

(4) How do perceived risks of being sexually harassed motivate protective behaviors among Zambian soldiers in selected military camps?

\subsection{Study Design}

\subsubsection{Hermeneutic Phenomenology Design}

Hermeneutic Phenomenology study design is justified because it was used to uncover the lived experiences regarding the link between perceived risks of being sexually harassed and protective behaviors adopted by Zambian soldiers. The Hermeneutic Phenomenology design is further justified because it further allowed the researcher to offer an interpretation to the study findings (Van-Manen 1990 in Carey 2012). The design is qualitative in nature and uses semi-structured interview guides inorder to gain knowledge. Perceived risk in this phenomenology study, which was based on the lived experiences was measured by examining the perspective of participants, for an explanation on whether they perceive; no risk, low risk or high risk of sexual harassment. Further lived experiences regarding the awareness of the potential risk factors were also explored. Lastly, experiences on whether those that perceived risk of being sexually harassed in terms of whether they were aware and/or adopted protective behaviors. The Self-Report Response Strategy based on semi-structured interviews was used as it not only allowed for further probes, but also allowed for the protection of the lived experiences from the participants.

The lived experiences demanded physical interviews because exploration of the link between perceived risk of being sexually harassed and protective behaviors among soldiers demanded the insiders view.

\section{The underpinning Ontology Philosophy supporting the Hermeneutic Phenomenology Study Design}

The Nominalist Ontology Philosophy was used as a solid foundation in strengthening of the Hermeneutic Phenomenology research design. This was largely because the Nominalist Ontology Philosophical implications are linked to the 'emic perspective', in which the 'Social Reality' that was 
being pursued was seen to be created in the minds of the researched. This therefore supported the design because the information that was needed was, also not tangible it was based on the latent thought processes of the researched (Burrell and Morgan in Carey, 2012). This therefore, supported (lived experiences) which are also based on latent thought processes of the participants under the phenomenology design.

The philosophy of science in the research considered the 'emic perspective' inorder to support the scientific research design which considered the 'lived experiences'.

The Nominalist Ontological question, showing the link between Perceived Risk of being sexually harassed and protective behaviours adopted by soldiers further required a philosophy that would theorise the production of such knowledge. The philosophy of science, which theorise production of knowledge in research is the epistemology which in this study was linked to the qualitative approach.

\subsubsection{Importance of Qualitative Approach in relation to the Hermeneutic Phenomenology Study Design}

The main thrust of the qualitative approach, within the scientific research process lies in the production of knowledge, from participant subjective meanings about a particular phenomenon within their natural settings using physical interviews. In this case the physical interviews in this study were tied to the Semi-Structured Interviews. Phenomenology is under the umbrella of the Qualitative Approach.

Perceived risk of sexual harassment and protective behaviors can be studied qualitatively or quantitatively. The reviewed literature in chapter two showed how perceived risk of sexual harassment was studied quantitatively by Fontalvo et al (2019). The reviewed literature in chapter two also showed how perceived risk of gender based violence was studied qualitatively by Kaufman et al (2019).

However, perceived risks of being sexually harassed and protective behaviors adopted by soldiers globally appear to be under explored, Zambia inclusive. An under explored phenomenon can effectively be studied using the qualitative approach which is also the main umbrella for phenomenology (Carey, 2012). Therefore, the qualitative approach which also embraces exploratory motives was useful because the concept of perceived risk of sexual and protective behaviors adopted by soldiers appear to lack prior knowledge. Further the justification for the use of the qualitative approach is because it is able to allow for follow ups to the experiences on the verbal ideas, responses as well as allowing for further investigation to motives and feelings about the phenomenon. This is done within the natural settings of the participants. The resultant observations or interviews lead to the identification of new emerging patterns and trends whose summaries may help in the revision or development of a new theory or proposals of statements. These in turn helped to understand and explain what was discovered. This is important for under explored phenomenon.

\section{The underpinning Epistemology Philosophy to the Qualitative Approach}

The Interprativist Epistemology Philosophy was used as a solid foundation in strengthening of the qualitative research approach. This because the Interprativist Epistemology Philosophical implications supported the knowledge generation, suggesting that 'there are no fixed truths' meaning the understanding and meanings participants regarding the perceived risk of sexual harassment among soldiers and their adopted protective behaviors were different. When viewed from the perspective of the interpretive epistemology philosophy the 'lack of no fixed truths in participants' is tied to subjective experiences of participants under the qualitative approach (Burrell and Morgan in Carey, 2012). .

The philosophy of science in the research, suggested that 'there are no fixed truths' when considering the 'emic perspective', inorder to support the qualitative research approach which also looked at participant subjective meanings about the 'lived experiences' of a particular phenomenon within their natural settings using physical interviews.

This because the generation of such knowledge is dependent on the subjective independent motives about how the participants felt about the phenomenon. The philosophy therefore supports the assertion that the knowledge about the phenomenon can be studied in the natural settings of the 
participants which in this case was the selected military areas. Further the philosophy supported the qualitative approach by confirming that the knowledge of the exploratory motive and probes about the problem can only be enhanced by assuring the individual participants of protection which was achieved through assurance of protection of anonymity and confidentiality. This was enhanced by the use of pseudonyms.

\subsection{Study Sites}

The study was conducted in three selected targeted military camps headed by field ranks of Ndola, Lusaka and Chipata. Large camps headed by field ranks are potential sexual harassment areas hence the choice of the selected areas were the camps are found (Foucault, 1975).

\subsection{Target Population}

Inclusion of Victims and non-victims of sexual harassment was important in the understanding of protective behaviours that soldiers use when they perceive risks of being sexually harassed. NonVictim perspectives are important as their lived experiences may be used to help both potential and actual victims at individual level.

\subsection{Sample Size}

(96) Participants both victims and non-victims of sexual harassment were initially meant to be selected by this research, with not more than seven participants in all the seven non-commissioned rank categories that define a soldier (1.Privates, 2. Lance Corporal, 3.Corporal, 4. Sergeant, 5. Staff Sergeant, 6.Warrant Officer Class Two and 7.One). Although (Patton 1990 in Holloway and Wheeler,1994) argues that no sample size guidelines exist in qualitative research, each of the categories of ranks was sufficient because it did not have more than seven participants which is the maximum number for the commencement of data saturation Guba and Lincoln (1994). After this no more new information came up and in depth understanding of a phenomenon were achieved Guba and Lincoln (1994). In the present research the data saturation was constant and was arrived at three participants that were selected from each category. This was because the categories of ranks had homogenous or similar characteristics (Carey, 2012). It was against that background that selection was then stopped for each particular category of rank so that analysis for the different findings take place (ibid, 2012). The principle of data saturation was first postulated by Barney Glasser and Anselm Straus in 1967 when they introduced grounded theory. The duo argued that data saturation is a strategy used in qualitative as a criterion for discontinuing data collection and/or analysis which may be achieved between 3-7 participants (Glasser and Strauss ,1967).

However, contemporary qualitative researchers such as Van Manen a Phenomenologist argued that the principal had limitations because there was a possibility that there could be backflow of data which may not be reached as a result of this discontinuation. He therefore suggested that it would be important to move forward and backwards to ensure that saturation had been achieved (Van-Manen 1990 in Carey 2012).

Therefore this study adopted the two principles inorder to ensure that data saturation from participants had really been attained, by going ahead through going forward and backward to confirm if there is and/no backflow of data. The study later confirmed that saturation was consistent at 3 participants.

(75) Victims were to represent both the male and female soldiers. 35 male victims were selected. Five participants represented each of the seven categories of ranks that define a Soldier. 40 female victims were earmarked for selection. Six participants represented each of the seven categories that define a soldier. However, the last category for the females had only four because few reach the last rank bringing a total of 74 victims.

However, the findings from the victims showed that the senior categories of warrant officer class one and two and staff sergeants only suffered sexual harassment in the lower ranks. For males it included the rank of sergeant. This meant that the ranks were to be excluded when considering the nonvictims.

Therefore, the junior categories of ranks that were included were private, lance corporal, and corporal for both female and male non-victim soldiers. Additionally, the rank of sergeant for females was included. This is because this category continued to suffer sexual harassment in the present ranks. 
Because of the precedence in terms of data saturation 3 participants were earmarked for each of the categories of junior ranks mentioned as shown hereunder:

(21) Non-victims were the selected to add to the sample size.9 male non-victims and 12 female nonvictims. Each category had not more than three which were all sufficient to attain data saturation hence the justification.

\subsection{Sampling Procedures}

All soldiers (Victims and Non Victims) were eligible. A Sexual Harassment Experience Questionnaire (SHEQ) was used to identify the participants based on their perceived risk situation, understanding of sexual harassment and its forms and the protective behavior situation. The instrument was supplemented by the purposeful sampling. Both techniques were used due to lack of an availed sampling frame for both categories of participants.

According to Fitzgerald et al (1999) the Sexual Experience Questionnaire (SEQ) was meant to come up with measurements for sexual harassment and is also used to establish the prevalence and frequency rates. The establishment of the prevalence rates then leads to the identification of those people that have been harassed from those that have not been harassed, based on the understanding of what constitutes sexual harassment.

The Sexual Harassment Experience Questionnaire (SHEQ) therefore becomes a stepping stone for an in-depth understanding of how the problem occurs based on the lived experiences by victim participants. This was to be achieved by interrogating subjective views using the semi-structured interviews or focus group discussion interviews.

Based on the validated Sexual Harassment Experience Questionnaire (SHEQ) roles the present study replication was justified.

The Sexual Harassment Experience Questionnaire (SHEQ) was therefore, used to identify the participants based on their perceived risk situation, understanding of sexual harassment and its forms and the protective behavior situation. This was achieved by random departmental visitations of the selected military camps until the desired sample was attained. Understanding of sexual harassment and its forms entailed that soldiers could perceive risk of sexual harassment and either come up with protective behaviors or not depending on their subjective judgments. Those that did not understand sexual harassment and its forms were excluded as they were not able to perceive the risk. Purposeful Sampling was used to select participants that met the criteria for this study based on the Sexual Harassment Experience Questionnaire (SHEQ). The participants that were selected from the camps came from the non-commissioned ranks that define a soldier which are (1.Privates, 2. Lance Corporal, 3.Corporal, 4.Sergeant, 5.Staff Sergeant, 6. Warrant Officer Class Two and 7. One) in ascending order from the junior to the senior most category of rank. Literature shows that this is the category that is affected (UNSC, 2008). Although, literature is silent on the commissioned ranks it is possible that they can also be affected. However, ethically the researcher being a soldier cannot study commissioned officers because of differential power relations. Therefore, the experiences of the present study can also be extrapolated to the officers.

\subsection{Data Collecting Procedure and Quality Assurance}

In this study information was collected from both secondary sources of data and primary sources of data.

Secondary data collection: In its quest to collect data related to the topic under this study, the researcher got the information from research text books, dissertations, theses and journal articles. Inorder to identify gaps leading to the identification of the methodology and methods used by this research, the narrative review of literature, which is the traditionally acceptable type within the qualitative research and most relied because it is a pragmatic and less structured approach was used (Hart, 2013).

The narrative type review of literature did not only help in the discovery of new insights about the topic but also helped in designing the research methodology and methods which is the third stage in a formal research process. 
Primary data collection: The study used the Semi-Structured Interview guides to collect raw data in the field. However, before they were used, they were subjected to some form of preliminary test inorder to establish whether the questions would yield the required information.

\subsubsection{Primary Data Collection and Quality Assurance}

Pretesting was used as a preliminary test involving baseline knowledge as well as preparedness for the research process which was done to ensure quality data is obtained (Creswell, 2012 and Carey, 2012). Guba's four trustworthy strategies which include Credibility, Dependability, Confirmability and Transferability were used inorder for the assurance of data quality as well as the study academic acceptability (Guba and Lincoln, 1994).

This was achieved in four stages during the data collection procedure.

\section{First stage of ensuring qualitative primary data collection is of good quality}

The first stage in ensuring that data collection was of good quality began with the identification and accurate description of potential participants. Inorder to identify the potential participants, in the absence of an availed sampling framework, the study made use of a researcher/self-administered Sexual Harassment Experience Questionnaire (SHEQ). Affirmative response toward the defined concepts the study under the definition of concepts section page (i) before the introduction through Sexual Harassment Experience Questionnaire (SHEQ) appendix 1 page (91) meant automatic purposeful selection. This was with regard to sexual harassment and perceived risk.

Further the potential participants were classified into two groups based on their response towards the question on the protective behavioral status also indicated under the definition of concepts section page (i) before the introduction through Sexual Harassment Experience Questionnaire (SHEQ) appendix 1.This was from the Non-commissioned ranks that define a soldier also indicated on the same pages. The accurate description of the participants was later facilitated by purposeful selection.

Based on the qualitative principles the researcher was assured of credible data through the use of a suitable design meant for the lived experiences which according to qualitative principles was the Hermeneutic Phenomenology and the Semi-Structured Interview Guide. The Semi-Structured Interview Guide was later subjected to a pretest in the second stage.

\section{Second stage of ensuring qualitative primary data collection is of good quality}

The Semi-Structured Interview Guide was then subjected to a pretest so that it had the capacity of having standardized questions that could easily be answered by the accurately defined participants.

However, during the preliminary test of the research instruments for standardization of questions, evidence showed that the researcher needed a female research assistant. This was noted from a few female participants who said they could only participate in the study if they were interviewed by a fellow female. Inorder, to ensure data quality and in-depth understanding of the topic a female researcher was identified and given research skills training.

This was to cater for few female participants that required a female inorder to open up. Research assistants therefore, came from a military background, with at least a first degree/research or better knowledge in a similar field. Further inorder to ensure data quality an age of thirty- five was appropriate for research assistants as it sits between youth and adulthood. The ideal rank was of staff sergeant who also fits the age category as well as service. Both old and young participants were able to freely express themselves freely in the few recorded cases. The duty of a female research assistant was to ensure that data that might be affected by virtue of the sex of the principal investigator is handled by her so that females can open up to ensure quality. Furthermore, inorder to ensure data quality the research assistant was guided on the prescribed interview process using the proposed research questions.

As a way to ensure further data was of quality participants were subjected to the self-report response strategy at individual level. They were told that this was going to ensure protection of the participants by remaining anonymous both before and after data collection which only involved the researcher and the individual participant. They were also told they would be identified by a pseudonym. As a result the participants were willing to open up. 
This made the researcher to be assured that he was going to collect dependable data during the process of actual data collection.

\section{Third stage of ensuring qualitative primary data collection is of good quality}

During the actual face to face interviews the researcher made sure that participant's data was secured by recording it the actual way in which it was reported. This was achieved by not going into the actual discussion with preconceived ideas that would influence data quality. This meant that data could be confirmed by other researchers. The information that came from the participants was in accordance with their lived experiences without interference from the researcher..

Therefore, the extent of the findings was authentic or valid meaning another researcher could get similar findings hence confirmable data quality was assured (Guba and Lincoln, 1994).

\section{Forth stage of ensuring qualitative primary data collection is of good quality}

Finally, after having considered the three preliminary qualitative principles for ensuring data was of quality the researcher assumed that it was now possible that final accumulated data could be transferable.

This means that the findings are now able to be viewed from a representative sample of the nonvictim and victims of sexual harassment to the population of Zambian soldiers. This means the work can be cited by other studies as well as be able to be contextualized with other academic studies because it has satisfied the preliminary information above

\subsubsection{Data Collecting Instruments}

Semi-Structured interview guides were used to collect data. It is an important tool as it is the most common form of interview in the qualitative research because it allows for pre-planned questions for participants to avoid losing focus. It also allows the researcher to come up with spontaneous questions or further probing to ideas, motives and feelings which are useful in under explored phenomenon. Further, using the formal face to face interview guide it is easy to get a depth understanding because the participants will be assured of confidentiality, anonymity and being untraceable during and after the study making it easier for them to open up (Carey, 2012).

\section{Interview Process to the research questions for both Pretesting and Actual Data Collection}

The researcher began each of the sessions with the individual victims and non-victims of sexual harassment for both male and female soldiers through a greeting. He went on to introduce himself. He told the participants that he was a student at the University of Zambia and that he had chosen the topic under discussion as a fulfillment of the requirements for the award of the Doctor of Philosophy Degree in Gender Studies because the topic captured his interest.

Before the interview session with each of the victim participants could commence, the researcher used the informed consent form to make the participants aware of the research and the procedures to be followed. He told the participants that the study was meant for academic purposes and that he wished to understand both the individual experiences of sexual harassment from a gendered perspective.

He told the participants that he wished to uncover there lived experiences on how Perceived Risk of sexual harassment is associated with protective behaviours from their verbal accounts of both nonvictim and victim soldiers in selected camps of Ndola, Chipata and Lusaka in Zambia.

Participants were further assured of confidentiality and that they were going to remain anonymous and untraceable in the research process thereby being addressed by a number and not by name, or pseudo name. The participants were then told that they were free to pull out at any point if they felt uncomfortable as it was there right during or before the session, which was voluntary.

They were then asked for a verbal or written consent. Those that gave consent in the affirmative were later administered with the Semi-Structured Interview guide using formal face to face questions.

He began with female and male victims before engaging female and male non-victims for all the four questions. 


\subsection{Data Analysis}

The study uses the Interpretive Phenomenology Analysis (IPA) alongside the Qualitative Risk Assessment or Analysis.

\section{a) Interpretive Phenomenology Analysis (IPA)}

The study used the Interpretive Phenomenology Analysis (IPA) because it is a methodological qualitative tool that is influenced by phenomenology, with a purpose of examining how people make sense of their major life or lived experiences. The Interpretive Phenomenology Analysis further draws its strength from the hermeneutics (the study of interpretation) (Smith et al, 2009).

The Interpretive Phenomenology Analysis is a powerful method for analyzing large amounts of Phenomological data collected from experiences of participants through semi-structured interviews or focus group discussions interviews (Smith and Osborne 2014). Further it has the capacity to condense raw data into categories based on valid inference and interpretation.

Based on the principles that fitted in the present study, this research was justified by adopting the Interpretive Phenomenology Analysis whose data presentation steps were replicated. There are three steps needed to be utilized since the primary data needed to be transcribed directly into verbal texts, which were adopted by the present research..

\section{The first stage (Sorting Stage)}

The first stage (Sorting Stage) began by arranging the interview protocols into the major themes by way of sorting them in the chronological order of the research questions. This lead to the order shown hereunder:

(1) How is the situation of perceived risk perceptions of being sexually harassed among Zambian soldiers in selected military camps?

(2) What risk factors are associated with sexual harassment among Zambian soldiers in selected military camps?

(3) What protective behaviors do Zambian soldiers adopt against the risk factors of being sexually harassed in selected military camps?

(4) How do perceived risks of being sexually harassed motivate protective behaviors among Zambian soldiers in selected military camps?

Having completed the sorting out stage the second stage involved reading of the interview protocols for the purpose of editing.

\section{The second stage (Editing Stage)}

The second stage (Editing Stage) meant going through each of the interview protocols by way of reading so as to edit the data. The researcher upon having sorted out the data from all the participants based on what had been spoken proceeded to read through each transcript. The choice of the content was justified by what the researcher desired to know. This is because the researcher needed the desired information that is going to be useful with regards to the objectives and research questions of the study. Having completed the editing stage the last stage will involve reading and transcribing of the interview protocols.

\section{The third stage (Coding Stage)}

The third stage (Coding Stage) involved coding of important concepts and constructs inorder to come up with understandable themes and sub themes by way of reading and transcribing the data into Verbatims that are easily understood based on what the participants had said. This was achieved by way of exploration of the relationship between patterns and trends through reading.

The researcher then transcribed the data from the desired form into Verbatims. These were reported in the main thesis. This is the last stage in the research process.

\section{b) Qualitative Risk Assessment or Analysis}

The study as already stated in the Preambo also used the Qualitative Risk Assessment or Analysis alongside the Interpretive Phenomenology Analysis. 


\section{First Step (Risk Identification)}

Qualitative Risk Assessment or Analysis was used to describe the overall process or method used to identify the threat or risk which was (sexual harassment) as well as potential risk factors that have a possibility of occurring (Risk Identification).

\section{Second Step (Risk Analysis)}

The overall risk analysis for sexual harassment of both non-victims and victims was calculated by multiplying the possibility with the severity, based on the balanced qualitative score card that had generic measurements low (1), medium (2) and high (3) predetermined ratings/levels.

\section{Third Step ( 3 by 3 Risk Analysis Matrix for sexual harassment).}

The risk matrix used two major components that included the (a) Possibility and (b) Severity in assessing and analyzing the risk of sexual harassment. Both components use used three standard colour codes which are Red (high), Orange(medium) and Green (low) in coming up with the overall qualitative risk analysis (Graves, 2000). The red colour was centered on the top right corner of the risk matrix. It represented high severity and high possibility for the occurrence of sexual harassment among soldiers. For example if the possibility on the left side of the boxes increasing upward is high (3) and the severity below the boxes increasing to the right is high (3) then the overall risk is (9). This means a high risk scale with a red colour is very likely to be given priority in terms of adoption of protective behavior in this case against the sexual harassment risk factors. Additionally, if the possibility on the left side of the boxes increasing upward is high (3) and the severity below the boxes increasing to the right is high (2) then the overall risk is (6). This also means a high risk scale with a red colour is likely to be given priority in terms of adoption of protective behavior in this case against the risk factors.

The green colour was centered on the bottom left corner of the risk matrix. It represented very low severity and very low possibility for the occurrence of sexual harassment among soldiers.

While the orange colour (low) was centred between the red colour (high) and green colour (very low).

The principal in all cases is the same the product of a risk $=$ Possibility by Severity as indicated above.

Vertical $(\mathrm{Y})$ Axis $=$ Horizontal $(\mathrm{X})$ Axis begins with 1. Low 2. Medium or 3.High increasing to the right.

\section{The underpinning Methodology Research Philosophy supporting the qualitative methods of collection and analysis of data}

The Idiographic Methodology Philosophy in this research was used as a solid foundation in strengthening of the qualitative methods. This was largely because its main thrust is to focus on the understanding of individual behavior from a small sample. The resultant emerging themes were established from the Perceived Risk of being sexually harassed and protective behaviours adopted that are adopted by soldiers. Small samples in the practice of this philosophy led to the easy analysis of the emerging themes.

The small sample that is aimed at understanding of individual behavior advocated by idiographic methodology philosophy supports the qualitative data saturation issues within the sample size which are tied to non-random selection methods under the qualitative research methods. For the qualitative approach the methods were further tied to physical interviews done by semi-structured interviews and the interpretive phenomenology analysis as shown under the methods (Burrell and Morgan, 1979; in Carey 20

\section{RESEARCH FINDINGS AND DISCUSSIONS}

\subsection{Research Questions}

(1) How is the situation of perceived risk of being sexually harassed among Zambian soldiers in selected military camps? 
(2) What risk factors are associated with sexual harassment among Zambian soldiers in selected military camps?

(3) What protective behaviors do Zambian soldiers adopt against the risk factors of being sexually harassed in selected military camps?

(4) How do perceived risks of being sexually harassed motivate protective behaviors among Zambian soldiers in selected military camps?

The participants were identified and accurately described through the researcher/self-administered Sexual Harassment Experience Questionnaire (SHEQ). Affirmative response toward the defined concepts of the study under the definition of concepts section page (i) before the introduction through Sexual Harassment Experience Questionnaire (SHEQ) appendix 1 page meant automatic purposeful selection. Since the participants accepted the definition the researcher is was confident that they would further give a credible in-depth understanding of the concepts of sexual harassment (physical, verbal and non-verbal) unwelcome actions and perceived risk (low, medium or high) awareness situation on question one.

Further since the participants were classified into two groups' non-victims and victims based on their response towards the question on the protective behavioral status an in-depth understanding was also yielded through question three and four. The accurate description of the participants was later facilitated by purposeful selection hence; credible data was assured through the semi-structured interview guide that made use of the prescribed interview process below.

\subsection{Interview Process to the Research Questions}

The researcher began each of the sessions with the individual victims and non-victims of sexual harassment for both male and female soldiers through a greeting. He went on to introduce himself. He told the participants that he was a student at the University of Zambia and that he had chosen the topic under discussion as a fulfillment of the requirements for the award of the Doctor of Philosophy Degree in Gender Studies because the topic captured his interest.

Before the interview session with each of the victim participants could commence, the researcher used the informed consent form to make the participants aware of the research and the procedures to be followed. He told the participants that the study was meant for academic purposes and that he wished to understand both the individual experiences of sexual harassment from a gendered perspective.

He told the participants that he wished to uncover there lived experiences on how Perceived Risk of sexual harassment is associated with protective behaviours from their verbal accounts of both nonvictim and victim soldiers in selected camps of Ndola, Chipata and Lusaka in Zambia.

Participants were further assured of confidentiality and that they were going to remain anonymous and untraceable in the research process thereby being addressed by a number and not by name, or pseudo name. The participants were then told that they were free to pull out at any point if they felt uncomfortable as it was there right during or before the session, which was voluntary.

They were then asked for a verbal or written consent. Those that gave consent in the affirmative were later administered with the Semi-Structured Interview guide using formal face to face questions.

He began with female and male victims before engaging female and male non-victims for all the four questions.

\section{Research Question One}

(1) How is the situation of perceived risk of being sexually harassed among Zambian soldiers in selected military camps?

\section{Thematic Sub Questions:}

- Please tell me about yourself.

- Kindly tell me your actual sexual harassment experience?

- In your own opinion as a (victim) explain what makes you Perceive (High or Medium or Low) Risk towards sexual harassment ..? 


\subsubsection{Research Findings to the Research Question One (General summary of findings).}

The study combined responses from both the victim and non-victim participants. This was with regards to the situation of perceived risk of being sexually harassed among Zambian soldiers in selected military camps. The study further went on to establish that most of female victims who were of the ranks of private, lance corporal, corporal and sergeant suffered from verbal and nonverbal sexual harassment. The study found out that the perpetrators were male soldiers within the same range of ranks but either senior by date of promotion or a step ahead. On the other hand, it was also established that the male victims of the ranks of private, lance corporal and corporal suffered from verbal sexual harassment. The study found out that the perpetrators were female soldiers within the same range of ranks but either senior by date of promotion or a step ahead. On the other hand female non-victim soldiers within the same category also affirmed the verbal and non-verbal sexual harassment. However, the non-victims confirmed that they do not suffer from sexual harassment affirmed existence of verbal/non-verbal sexual harassment. The male and female non-victim soldiers in this category also affirmed the verbal sexual harassment. There was sufficient combined individual evidence from both victims and non-victims showing that they experienced highperceived or awareness towards risk of being sexually harassed. This came from both male and female soldiers.

Sub-Theme Number One: Physical Experiences Of Sexual Harassment: Male and Female Victim Verbatims.

According to a Female Victim soldier of the Rank of Private by the name of Josephine Mtonga, Josephine Phiri, Josephine Daka, Lance Corporal by the name of Pascalina Chilongoshi, Amina Abili, Amina Ability, Corporal by the name of Jeannie Shapa, Sheba Babie, Janet Shapa Sergeant by the name of Joyce Kalebwe, Joyce Mulemwa and Joyce Mulemwa (Pseudo 'not real' Names) when asked on thePhysical Experiences Of Sexual Harassment among the interaction of soldiers in the Non-Commissioned Ranks Individually and severally said that:

"Thank very much. Having told you how I understand sexual harassment I now wish to talk about my story.......... Sir (meaning the researcher) just like I said earlier on regarding my understanding of sexual harassment that it is any action from a man that is not wanted by a woman.... I wish to say that I have experienced it through an unwanted action. Sir (meaning the researcher) during my training, a male instructor when marching walked behind saying in Chinyanja that 'Iwe msikali wazulisa combat yatu kumbuyo', meaning soldier your combat trouser is full at our behind. That to me was verbal sexual harassment because I did not approve it knowing that it could lead to other things.... Another one told me that you females have come to destroy the system as there is no female buffalo representing

Male Victim Soldier of the Rank of Private by the name of Thomson Kunda, Private Rodrick Palula, Antony Phiri, Lance Corporal by the name of Alexander Msiska, Stanley Chipowe, Floyd Kamanga, Corporal Abraham Mubita, George Muyeba and James Banda (Pseudo 'not real' Names) when asked on the Physical Experiences Of Sexual Harassment among the interaction of soldiers in the Non-Commissioned Ranks Individually and severally said that:

"I have been a victim of sexual harassment I am always a victim. I wish to state that my physical experience that I encounter with regard to the problem is verbal sexual harassment, which was in form of unwanted comments. Sir (meaning the researcher), as a junior non-commissioned rank, I suffer unwanted comments if I am beaten in class or at the morning run by senior female soldiers of the ranks of Staff Sergeant, Warrant Officer Class Two and One. They tell me that I am a woman...."

It must also be noted that the remaining category of ranks for both males and females victims also reported that physical sexual harassment came in form of both verbal and non-verbal from senior ranks among their interactions. However, the categories further added that these physical experiences were not in their present ranks but in the similar ranks who's Verbatims have since been recorded.

Sub-Theme Number Two: In your own opinion as a (victim) explain what makes you Perceive (High, Medium or Low) Risk towards sexual harassment: Male and Female victim verbatim

According to a Female Victim soldier of the Rank of Private by the name of Josephine Mtonga, Josephine Phiri, Josephine Daka, Lance Corporal by the name of Pascalina Chilongoshi, Amina Abili, 
Amina Ability, Corporal by the name of Jeannie Shapa, Sheba Babie, Janet Shapa Sergeant by the name of Joyce Kalebwe, Joyce Mulemwa and Joyce Mulemwa (Pseudo 'not real' Names) when asked to explain what makes her to Perceive (High or Medium or Low) Risk towards sexual harassment among the interaction of soldiers in the Non-Commissioned Ranks Individually and severally said that:

"Sir, 'meaning the researcher' I am currently a victim of sexual harassment and I must tell you that I have a high perceived or awareness towards risk of being sexually harassed because I have never heard of any defined prevention strategies even though we have a gender desk at Army Headquarters. These military characteristics put me at high risk. However, I will be lying to say I have not heard of any prevention strategies.................... In 2016 a team of Zambia National Service Gender persons led by a Lieutenant Colonel talked about the types of sexual harassment and verbally said it would be important to report the harassers to bosses.................. Furthermore, the characteristics of those that are senior or perpetrators make my perceived risk to be high .........................."

According, to a Male Victim Soldier of the Rank of Private by the name of Thomson Kunda, Private Rodrick Palula, Antony Phiri, Lance Corporal by the name of Alexander Msiska, Stanley Chipowe, Floyd Kamanga, Corporal Abraham Mubita, George Muyeba and James Banda (Pseudo 'not real' Names) when asked to explain what makes him to Perceive (High or Medium or Low) Risk towards sexual harassment among the interaction of soldiers in the Non-Commissioned Ranks Individually and severally said that:

"Sir 'meaning the researcher 'I always come under unwanted actions that are verbal in nature as I have already said........................... but I have not come across any counseling military authorities so that the problem is stopped..... because of this I perceive high risk of being verbally sexually harassed..... another thing that makes me to have high perceived or awareness towards the risk is based on my personal weakness such as fearing to report the harassers as well as having senior female soldiers that are abusive... ,"

The ranks between sergeant to warrant officer class one for both male and female soldiers said they also perceived high risk of sexual harassment but not in their present ranks. Their verbatim are similar to the categories above rank categories. This means the category does not also suffer sexual harassment. It is against this background the category was excluded when dealing with the nonvictim category.

Further since the data saturation was consistent due to the homogenous characteristics which was ending at three participants each non-victim category of rank was represented by three participants.

\section{Sub-Theme One:}

\section{Physical Experiences of Sexual Harassment: Male and Female Non-Victim Verbatims.}

According to an individual Female 'Non- Victim' Soldier of the Rank of Private by the name Judy Musoko, Joyce Musenge, Tizzy Lemba Lance Corporal by the name of Patricia Chosi, Chonya Amasi, Chama Abby, Corporal by the name of Chisoni Musonda, Chola Stella, Chipo Stella Sergeant by the name of Aggie Musonda, Chilila Stella, and Chilekwa Stella (Pseudo 'not real' Names) when asked on the Physical Experiences of Sexual Harassment among the interaction of soldiers in the NonCommissioned Ranks individually and severally had this to say:

"Madam meaning the 'the female research assistant' I am very happy to be given another chance to provide an answer on myphysical experiences regarding sexual harassment ......................... Having initially told you that I do understand sexual harassment as any unwanted verbal or non-verbal action from a senior male soldier I wish to state that I have not experienced it.

According to an individual Male 'Non-Victim' Soldier of the Rank of Private by the name of James Muko, Joe Senge, Taiza Lembani, Lance Corporal by the name of Joseph Chisi, Chonya Mwenya, Chama Chanda, Corporal by the name of Chisoni Musonda, Chewe Musonda, Chola Stanley and Chola Chitalu (Pseudo 'not real' Names) when asked onthePhysical Experiences of Sexual Harassment among the interaction of soldiers in the Non-Commissioned Ranks individually and severally had this to say: 
Perceived Risk of Sexual Harassment and Protective Behaviours among Zambian Soldiers in Selected Military Camps

"Sir meaning the 'researcher' regarding myphysical experiences for sexual harassment...................... Although I understand sexual harassment as any unwanted verbal action from a senior female soldier $I$ wish to state that $I$ have not experienced it ................................................. from any female because I have ways of avoiding these people..... ,

Sub-Theme Number Two: In your own opinion as a (victim) explain what makes you Perceive (High or Medium or Low) Risk towards sexual harassment: Female non-victim verbatim

According to an individual Female 'Non- Victim' Soldier of the Rank of Private by the name Judy Musoko, Joyce Musenge, Tizzy Lemba Lance Corporal by the name of Patricia Chosi, Chonya Amasi, Chama Abby, Corporal by the name of Chisoni Musonda, Chola Stella, Chipo Stella Sergeant by the name of Aggie Musonda, Chilila Stella, and Chilekwa Stella (Pseudo 'not real' Names) when asked on what makes you Perceive (High or Medium or Low ) Risk in the Non-Commissioned Ranks individually and severally had this to say:

'Sir, 'meaning the researcher' I am not a victim of sexual harassment but I must tell you that I have a high perceived or awareness towards the risk of being sexually harassed because I have never heard of any defined general prevention mechanisms.... These military characteristics put me at high risk. However, I will be lying to say I have not heard of any prevention strategies...................... Even though there appear to be high risk of being sexually harassed I use my own initiative to protect myself........Furthermore, the characteristics of those that are senior or perpetrators make my perceived risk to be high

According to an individual Male 'Non-Victim' Soldier of the Rank of Private by the name of James Muko, Joe Senge, Taiza Lembani, Lance Corporal by the name of Joseph Chisi, Chonya Mwenya, Chama Chanda, Corporal by the name of Chisoni Musonda, Chewe Musonda, Chola Stanley and Chola Chitalu (Pseudo 'not real' Names) when asked onwhether they perceived risk (high or medium or low ) risk regarding the risk of sexual harassment, they individually and severally said that:

"Sir 'meaning the researcher 'I am aware that some of my friends are harassed because of lack of any counseling military authorities so that the problem is stopped..... because of this I also perceive or awareness that is high towards the risk of being verbally sexually harassed..... however, I manage to protect myself...

\section{Studies outside Africa (Similarities).}

A study conducted by Gallagher (2008), in the United States of American Army also found that the type of sexual harassment among the female officer cadets under training was verbal and nonverbal. The findings of the study are similar with those for the present inquiry in the selected military camps of Zambia.

The reason for the similarity in the findings between the present inquiry and the American study is because the military characteristics for the harassers and the victims may be similar across other Armies.

Furthermore, another study by Davidson (2013) in the United States of American Army also found that the type of sexual harassment among the interactions its service personnel was high and that the type of sexual harassment was verbal. In the American study the victims of sexual harassment were female military personnel.

The reason for the similarity in the findings of the American study with the Zambian study may be alluded to the fact that the characteristics of the of the male harassers might be in one way or the other similar due to the upbringing in terms of the way they are trained.

Furthermore, Panday (2008) in the Indian Army, established that the hostile work environment referred to unwelcome sexual, advances, vulgar language and requests for sexual favours or other verbal and non-verbal conduct which interferes with work performance.

The reason for the similarity in findings is that the female victims in both the Indian and the Zambian study may have similar characteristics that lead to being harassed. Furthermore, the reasons for also not recording prevention strategies might be ascribed to the uniform way of management of military personnel by military commanders. 
Furthermore, Buchanan (2014) in the United States Army established that the most common type of sexual harassment was the gender harassment which involved unwelcome verbal and non-verbal actions from senior military ranks.

The reason for the similarity in findings may be ascribed to the fact that both studies had participants or victims who are female that may have similarities in the way females interact with the male senior ranked personnel at different levels. It may be further stated that reason for the findings on the females could be that the males discriminatory behavior that makes try to come up with unwanted comments on the victims.

Another study conducted by Pryor in (2010) having considered dimensions, and correlates of psychosocial harm that women in the military faced he went ahead to conduct a retest they concluded that behavioral domain with respect to physical, verbal and non-verbal unwelcome actions of sexual harassment fell in three major classes. These included sexual coercion, gender harassment and unwanted sexual attention in the military communities among soldiers.

The reason for the similarity in findings is that the female victims in both the United States and the Zambian study may have similar characteristics that lead to being harassed. Furthermore, the reasons for also not recording prevention strategies might be ascribed to the uniform way of management of military personnel by military commanders.

A study conducted by Fitzgerald (et al 1999) established that Gender harassment referred to as a broad range of verbal and non-verbal behaviors that were not aimed at sexual cooperation but those which conveyed insulting, hostile, and degrading attitudes affected female soldiers. Some elicited examples included threatening, intimidating or hostile acts that are all unwelcome.

The reason for the similarities in findings is ascribed to the fact that the military culture is some similar with only minor variations.

\section{Studies within Zambia (Similarities)}

A landmark study by Matakala (2015) that was conducted in Ndola with female soldiers found out that the type of sexual harassment for female soldiers was verbal and nonverbal sexual harassment among the interactions of its service personnel was also high. The verbal findings were similar to the present study. For example

The reason for the similarity in this finding is because both studies targeted Non-Commissioned Ranks for soldiers - 1.Private, 2.Lance Corporal 3. Corporal, 4.Sergeant, 5.Staff Sergeant, 6.Warrant Officer Class Two and 7.One in ascending order from the junior to the highest rank in this category. These have similar characteristics in terms of the way they interact.

It may also be assumed that the females in both studies are associated with a highly patriarchal structure that may be similar in terms of the characteristics of the male harassers.

Furthermore, another study by Matakala e'tal (2018) that was conducted in Zambia among soldiers came up similar findings that the type of sexual harassment was verbal and nonverbal for females based on the gendered perspective of the interactions of the soldiers. The verbal findings were similar to the present study.

For example on (page 11) a female soldier said "Senior male soldiers tell us in Nyanja that 'imwe bakazi kulibe buffalo ikazi mwabwela kuononga nchito meaning in English that There is no female buffalo in our system you females have come to destroy our work."

Both Zambian studies that were conducted by the aforementioned scholar are similar to the present study. This is because the present inquiry also found out from both male and female soldiers that unwanted actions that were verbal and nonverbal.

The reason for the similarity in the findings is because it is ascribed that the cultural aspects in terms of the behaviors of the participants that are similar in the military interactions for both the males and females are the same. Further the similarity may be ascribed to similar way of administration.

Discussion Of Research Findings To The Research Question One (Dissimilarities with other reviewed literature) (New Knowledge) 
There was sufficient combined individual evidence from non-victims showing that they experienced high perceived risk of being sexually harassed. This came from both male and female soldiers. This situation regarding the high perceived risk was ascribed to individual, sexist/perpetrator and military potential risk factors.

The reason for the new knowledge is assumed that an interaction with literature seem to have shown no study other study that looked non-victims. This is with regard to the situation of high perceived risk that was ascribed to individual, sexist/perpetrator and military potential risk factors.

Therefore the study as indicated under the significance is important because it has given an insight into the different perceive risks of sexual harassment for soldiers in selected military camps.

\section{Research Question Number Two (02).}

(2) What risk factors are associated with sexual harassment among Zambian soldiers in selected military camps?

\section{Thematic Sub Questions:}

- How do you think sexist/perpetrator/sexual harassers contribute to the potential risks that could increase sexual harassment among the interaction of soldiers?

- What do you think are the major individual victim potential risk factors that are associated with the occurrences of sexual harassment?

- In your own opinion describe the military potential risk factors that you think are associated with the occurrences of sexual harassment.

\subsubsection{Research Findings To The Research Question Two (General summary of findings).}

\section{Research Question Number Two}

(2) What risk factors are associated with sexual harassment among Zambian soldiers in selected military camps? The study combined responses from both the victim and non-victim participants.

This was with regards to the potential risk factors that are associated with sexual harassment among Zambian soldiers in selected military camps.

The study findings showed that both the male and female victim/non-victim soldiers showed that there were three major ways in Sexist/Perpetrator/Sexual Harassers as a first of the potential risks factors increases sexual harassment among the interaction of soldiers.

Bully, Discriminatory and Stalking characteristics were established as the three major classifications in which senior male and senior female Sexist/Perpetrator/Sexual Harassers contributed to the potential risks that increased sexual harassment among the interaction of soldiers.

The study further went on to establish that female and male victims had one major way in which they thought individual potential risk factors increases sexual harassment among the interaction of soldiers. This was through Non-Reporting of the senior male and female Sexual Harassers to relevant authorities by victims hence being the major contributor to the occurrences of sexual harassment among the interaction of soldiers. However, the non-victims were not asked on the second sub-theme as they knew the characteristics of the harassers and said they were not harassed hence the exclusion from the question on how individual potential risk factors may increase sexual harassment.

Lastly, the study found out from both the male and female victim/non-victim soldiers that there were three major ways in which military potential risk factors increases sexual harassment among the interaction of soldiers. Obedience and Discipline as (Military Culture), Lack of written military prevention measures against sexual harassment and Differential seniority in rank structure were the three major ways in which military potential risk factors increases sexual harassment among the interaction of soldiers.

Theme Number One: Sexist/Perpetrator/Sexual Harassers contributing to the potential risks that could increase sexual harassment among the interaction of soldiers: Male and Female Victim Verbatims. 


\section{Bully Senior Male Harasser Potential Risk Characteristics (Sub-Theme One).}

According to an individual Female 'Victim' Soldier of the Rank of Private by the name of Josephine Mtonga, Josephine Phiri, Lance Corporal by the name of Pascalina Chilongoshi, Amina Abili, Corporal by the name of Jeannie Shapa, Sheba Babie, Sergeant by the name of Joyce Kalebwe, Brenda Kaloshi and Joyce Mulemwa (Pseudo 'not real' Names) when asked onthe Sexist/Perpetrator/Sexual Harassers contributing to the potential risks that could increase sexual harassment among the interaction of soldiers in the Non-Commissioned Ranks individually and severally had this to say:

"Having chatted with you was at individual level last time ... Once again I am very happy to be given a chance to provide an answer on howSexist/Perpetrator/Sexual Harassers contribute to the potential risks that could increase sexual harassment .......... This is when a senior man becomes a bully because they will keep on doing this verbally or non-verbally knowing that the junior will not do anything about it. It happens to me every time....

\section{Discriminatory Senior Male Harasser Potential Risk Characteristics (Sub-Theme Two).}

Another Individual Female 'Victim', Soldier of the Rank of Private by the name of Josephine Phiri Lance Corporal by the name of Pascalina Chilongoshi, Amina Abili, Corporal by the name of Jeannie Shapa, Sheba Babie, Sergeant by the name of Joyce Kalebwe and Joyce Mulemwa (Pseudo 'not real' Names) when asked on the Sexist/Perpetrator/Sexual Harassers contributing to the potential risks that could increase sexual harassment among the interaction of soldiers in the Non-Commissioned Ranks Individually and severally had this to say:

"I wish to thank for allowing me to Providing an answer onthe Sexist/Perpetrator/Sexual Harassers contributing to the potential risks that could increase sexual harassment among the interaction of soldiers........... as a victim it is important to state that the male seniors keep saying that there is no female buffalo as can be seen from the Zambian army symbol........ They verbally say this to me and I can say this is discrimination of it highest level...............they normally say these words to me because they are senior.................... now know that each time I meet him he will say the words to me......

\section{Stalking Senior Male Harassers Potential Risk Characteristics (Sub-Theme Three).}

Furthermore, another individual Female 'Victim', Soldier of the Rank of Private by the name of Josephine Mtonga and Josephine Daka, Lance Corporal by the name of Amina Ability, Corporal by the name of Janet Shapa (Pseudo 'not real' Names) when asked on the Sexist/Perpetrator/Sexual Harassers contributing to the potential risks that could increase sexual harassment among the interaction of soldiers in the Non-Commissioned Ranks Individually and severally commented that:

"Sexist/Perpetrator/Sexual Harassers contribute to the potential risk that could increase sexual harassment among the interaction of soldiers through catching me unaware verbally and nonverbally especially when I am alone..... .".

The next category looks at the male victims.

\section{Bully Senior Female Harassers Characteristic Identification (Sub-Theme One).}

According to Male (Victim) soldier of the Rank of Private by the name of Thomson Kunda and a Lance Corporal by the name of Stanley Chipowe and Corporal by the name of Abraham Mubita (Pseudo 'not real' Names) when asked on the Sexist/Perpetrator/Sexual Harassers contributing to the potential risks that could increase sexual harassment among the interaction of soldiers in the NonCommissioned Ranks individually and severally said that:

'Sir meaning the 'researcher' Once again I am very happy to be given another chance to provide an answer on howSexist/Perpetrator/Sexual Harassers contributes to the potential risks that could increase sexual harassment among the interaction with the some senior females soldiers

I want to say that our senior female always want to take advantage of us junior ranks verbally

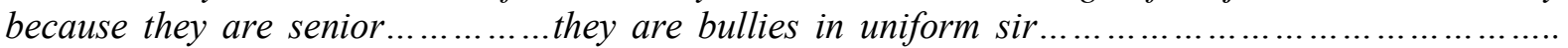
they just want to take advantage of me because of being senior"

\section{Inferiority Complex Senior Female Harassers Characteristic Identification (Sub-Theme Two).}


Another Male (Victim) soldier of the Ranks of Private by the name Thomson Kunda, Rodrick Palula, Antony Phiri Lance Corporal by the name of Alexander Msiska, Stanley Chipowe, Floyd Kamanga, Corporal by the name of George Muyeba, James Banda and Abraham Mubita (Pseudo 'not real' Name) when asked on Sexist/Perpetrator/Sexual Harassers contributing to the potential risks that could increase sexual harassment among the interaction of soldiers in the Non-Commissioned Ranks Individually had this to say:

'Sir meaning the 'researcher' Am happy to once again be given another chance to speak ....... This time around on my personal view concerning howSexist/Perpetrator/Sexual Harassers contribute to the potential risks that could increase sexual harassment..... I become aware of the threat of sexual harassment among the interaction with the females. I wish to say that most of the senior females have a feeling of being less important to me as a male.... And because of that they have different verbal actions that are not wanted by myself ............ When they say something they will not want to get an opinion from a man who is junior even if it is correct............ They will want to come up with faults as revenge because of this feeling on the junior men.....................Sir they suffer from inferiority complex.....

Theme Number Two: Individual victim potential risk factors that are associated with the occurrences of sexual harassment among the interaction of soldiers: Male and Female Victim Verbatims.

Non-Reporting of the senior male Sexual Harassers to relevant authorities by victims was said to be the major contributor to the occurrences of sexual harassment (Sub- Theme One)

According to an individual Female 'Victim' Soldier of the Rank of Private by the name of Josephine Mtonga , Josephine Phiri, Josephine Phiri, Lance Corporal by the name of Pascalina Chilongoshi, Amina Abili, Amina Ability, Corporal by the name of Jeannie Shapa, Sheba Babie, Janet Shapa Sergeant by the name of Joyce Kalebwe, Brenda Kaloshi and Joyce Mulemwa (Pseudo 'not real' Names) when asked onthe Sexist/Perpetrator/Sexual Harassers contributing to the potential risks that could increase sexual harassment among the interaction of soldiers in the NonCommissioned Ranks individually and severally had this to say:

"Having chatted with you initially, as an individual as well as a victim I feel that I am responsible for the occurrence of sexual harassment................................i say so because each time I am sexually harassed by the senior male harassers I don't report the issue to the relevant authorities for personal reasons.

Non-Reporting of the senior Male Sexual Harassers to relevant authorities by male victims was said to be the major contributor to the occurrences of sexual harassment (Sub-Theme One)

According to an individual Male 'Victim' Soldier of the Rank of Private by the name Thomson Kunda, Rodrick Palula, Antony Phiri, Lance Corporal by the name of Alexander Msiska, Stanley Chipowe, Floyd Kamanga, Corporal by the name of George Muyeba, James Banda, Abraham Mubita (Pseudo 'not real' Names) when asked onthe Individual potential risk factors that are associated with the occurrences of sexual harassment among the interaction of soldiers in the NonCommissioned Ranks individually and severally had this to say:

“......... each time I am sexually harassed by the senior male harassers I don't report the issue to the relevant authorities for personal reasons.................... is because I fear that my friends will laugh at me ..............................Senior ranks will laugh at me...............it is a mockery and unheard for a man to report a woman hence I fear.

Theme Number Three: military potential risk factors that you think are associated with the occurrences of sexual harassment: Male and Female Victim Verbatims.

Obedience and Discipline as [Military Culture], (Sub-Theme One).

According to an individual Female 'Victim' Soldier of the Rank of Private by the name of Josephine Mtonga, Josephine Phiri, Josephine Phiri, Lance Corporal by the name of Pascalina Chilongoshi, Amina Abili, Amina Ability, Corporal by the name of Jeannie Shapa, Sheba Babie, Janet Shapa Sergeant by the name of Joyce Kalebwe, Brenda Kaloshi and Joyce Mulemwa (Pseudo 'not real' Names) when asked onthe military potential risks that could increase sexual harassment 
among the interaction of soldiers in the Non-Commissioned Ranks individually and severally had this to say:

I am very happy to be given a chance to talk about military potential risk factors that could lead to the occurrence of sexual harassment...... I have a high a strong perception that because the military culture that is characterized with obedience and discipline especially for me as junior to my seniors leads to occurrences of sexual harassment............ This is because...... We are not supposed to say anything against a senior person whether they are right or wrong a senior person is always right................................................ours $i s$ to obey........because discipline is a culture

\section{Lack of written military prevention measures against sexual harassment (Sub-Theme Two).}

Another individual Female 'Victim' Soldier of the Rank of Private by the name of Josephine Phiri, Lance Corporal by the name of Pascalina Chilongoshi, , Corporal by the name of Jeannie Shapa, Sheba Babie, Janet Shapa Sergeant by the name of Joyce Kalebwe, Brenda Kaloshi and Joyce Mulemwa (Pseudo 'not real' Names) when asked onthe military potential risks that could increase sexual harassment among the interaction of soldiers in the Non-Commissioned Ranks individually and severally had this to say:

"Am happy to be asked once again on my view this time around concerning thereasons for military potential risk factors and how it leads to the occurrence of sexual harassment. I wish to say that am at a high risk to the exposure of sexual harassment because the harassers can't be afraid ................... The reason they are not afraid is because there appear to be no written prevention measures by the relevevant authorities ..............I think may be because the gender desk at the headquarters is not established yet as it has little manpower...."

\section{Differential seniority in rank structure (Sub-Theme Three).}

Furthermore, another individual Female 'Victim' Soldier of the Rank of Private by the name of Josephine Mtonga, Josephine Phiri, Josephine Phiri, Lance Corporal by the name of Pascalina Chilongoshi, Amina Abili, Amina Ability, Corporal by the name of Jeannie Shapa, Sheba Babie, Janet Shapa (Pseudo 'not real' Names) when asked onthe military contributing to the potential risks that could increase sexual harassment among the interaction of soldiers in the NonCommissioned Ranks individually and severally commented that:

"Strict differential seniority of ranks ......... is a tradition that results in the major occurrences of sexual harassment.......those in the higher ranks are the only ones that can be safe.....

This is because they hold higher ranks..........For me as a smaller rank it is very easy for me to be harassed"

The next category is that of the male victims.

\section{Obedience and Discipline as [Military Culture], (Sub-Theme One).}

According to an individual Male 'Victim' Soldier of the Rank of Private by the name of Thomson Kunda, Rodrick Palula, Antony Phiri, Lance Corporal by the name of Alexander Msisika, Stanley Chipowe, Floyd Kamanga, Corporal by the name of George Muyeba, James Banda and Abraham Mubita (Pseudo 'not real' Names) when asked onthe military potential risks that could increase sexual harassment among the interaction of soldiers in the Non-Commissioned Ranks individually and severally had this to say:

"I am very happy to be given a chance to talk about military potential risk that are linked to being sexually harassed by some senior female verbal sexual harassers...... Most of them are in a tendency of bullying me ............ This is because...... We are not supposed to say anything against a senior person whether they are right or wrong a senior person is always right, ours is to obey, because discipline is a culture in our system.

\section{Lack of written military prevention measures against sexual harassment (Sub-Theme Two).}

Another individual male victim soldier of the rank of lance corporal Floyd Kamanga (Pseudo "not real' Names) when asked onthe military potential risks that could increase sexual harassment among the interaction of soldiers in the Non-Commissioned Ranks individually and severally had this to say: 
"I think for me personally I feel there are no written military prevention statues against sexual harassment ..............................................that is the more reason women that are senior to us the men ..............due to inferiority complex they end up verbally abuse us.....

Theme Number One: Sexist/Perpetrator/Sexual Harassers contributing to the potential risks that could increase sexual harassment among the interaction of soldiers : Male and Female non-victim Verbatims.

\section{Bully Senior Male Harasser Potential Risk Characteristics (Sub-Theme One).}

According to an individual Female 'Non- Victim' Soldier of the Rank of Private by the name of Judy Musoko, Joyce Musenge, Tizzy Lemba, Lance Corporal by the name of Patricia Chosi, Chonya Amasi, Chama Abby, Corporal by the name of Chisoni Musonda,Chola Stella, Chipo Stella, Sergeant by the name of Aggie Musonda, Chilila Stella, Chilekwa Stella (Pseudo 'not real' Names) when asked onthe Sexist/Perpetrator/Sexual Harassers that may contribute to the potential risks that could increase sexual harassment among the interaction of soldiers in the Non-Commissioned Ranks individually and severally had this to say:

"Once again I am very happy to be given a chance to provide an answer on howSexist/Perpetrator/Sexual Harassers contribute to occurrence of sexual harassment through the unwanted behaviour of some senior male soldiers...........I see that these people always want to take advantage of those in the junior ranks verbally.............i see and hear this happening to my friends.....

\section{Discriminatory Senior Male Harasser Potential Risk Characteristics (Sub-Theme Two).}

Another Individual Female 'Non-Victim', Soldier of the Rank of Corporal by the name of Chisoni Musonda, Chola Stella, Chipo Stella, Sergeant by the name of Aggie Musonda, Chilila Stella, Chilekwa Stella (Pseudo 'not real' Names) when asked on the Sexist/Perpetrator/Sexual Harassers may contribute to the potential risks that could increase sexual harassment among the interaction of soldiers in the Non-Commissioned Ranks Individually and severally had this to say:

"Sexist/Perpetrator/Sexual Harassers contribute to the potential risks that could increase sexual harassment because I have noticed that some senior male are in the habit of telling junior women that there is no female buffalo on the military logo......... I say so because one of the victims told me so ......................................"

\section{Stalking Senior Male Harassers Potential Risk Characteristics (Sub-Theme Three).}

Furthermore, another individual Female 'Non-Victim', Soldier of the Rank of Private by the name of Judy Musoko, Joyce Musenge, Tizzy Lemba, (Pseudo 'not real' Names) when asked on the Sexist/Perpetrator/Sexual Harassers contributing to the potential risks that could increase sexual harassment among the interaction of soldiers in the Non-Commissioned Ranks Individually and severally commented that:

"Sexist/Perpetrator/Sexual Harassers contribute to the potential risk that could increase sexual harassment through unwanted actions were senior males are said to be targeting junior women soldiers that are alone with unwanted actions.............. have information from a friend of mine ".

\section{Bully Senior Female Harassers Characteristic Identification (Sub-Theme One).}

According to Male (Non-Victim) soldier of the Rank of Private by the name of Taiza Lembani, Corporal by the name of Chola Chitalu (Pseudo 'not real' Names) when asked on the Sexist/Perpetrator/Sexual Harassers contributing to the potential risks that could increase sexual harassment among the interaction of soldiers in the Non-Commissioned Ranks individually and severally said that:

"I have heard that there are some senior female soldiers who are in the habit of using there seniority to verbally sexually harass male juniors............ I know them and I also know there characteristics so that it is even easy for me to avoid them....." 


\section{Inferiority Complex Senior Female Harassers Characteristic Identification (Sub-Theme Two).}

Another Male (Non- Victim) soldier of the Ranks of Private by the name James Muko, Joe Senge, Taiza Lembani Lance Corporal by the name of Joseph Chisi, Chonya Mwenya, Chama Chanda, Corporal by the name of Chewe Musonda, Chola Stanley, Chola Chitalu (Pseudo 'not real' Name) when asked on Sexist/Perpetrator/Sexual Harassers contributing to the potential risks that could increase sexual harassment among the interaction of soldiers in the Non-Commissioned Ranks Individually had this to say:

'Sir meaning the 'researcher' I must tell you that senior female soldiers always have a negative attitude on me because I am a man............... They think I downplay them as women hence they end up harassing me verbally due to that reason......... I don't know where this inferiority complex comes from ........................................ Because I am aware of these characteristics I am able to tactically avoid them

Theme Number Two: military potential risk factors that you think are associated with the occurrences of sexual harassment : Male and Female Non-victim Verbatims.

\section{Obedience and Discipline as [Military Culture], (Sub-Theme One).}

According to an individual Female 'Non-Victim' Soldier of the Rank of Private by the name of Judy Musoko, Tizzy lemba, Lance Corporal by the name of Patricia Chosi (Pseudo 'not real' Names) when asked onthe military potential risks that could increase sexualharassment among the interaction of soldiers in the Non-Commissioned Ranks individually and severally had this to say:

"I am very happy to be given a chance to talk about military potential risk factors that could lead to the occurrence of sexual harassment...... In the military I am not supposed to answer back because that is regarded as indiscipline ................... Since I am not supposed to answer back I know this may lead to harassment............... However the good part is that I know the characteristics of the harassers hence I find ways of avoiding being harassed.....

Lack of written military prevention measures against sexual harassment (Sub-Theme Two).

Another individual Female 'Non- Victim' Soldier of the Rank of Private by the name Judy Musoko, Joyce Musenge, Tizzy Lemba Lance Corporal by the name of Patricia Chosi, Chonya Amasi, Chama Abby, Coporal by the name of Chisoni Musonda, Chola Stella, Chipo Stella Sergeant by the name of Aggie Musonda, Chilila Stella, and Chilekwa Stella (Pseudo 'not real' Names) when asked onthe militsry potential risks that could increase sexual harassment among the interaction of soldiers in the Non-Commissioned Ranks individually and severally had this to say:

"Am happy to be asked once again ........ there are no written measures on prevention of sexual harassment hence some of my friends being harassed........... For me lackly I know the characteristics of these people hence avoiding being a victim....

\section{Lack of written military prevention measures against sexual harassment (Sub-Theme One).}

According to an individual male 'Non-Victim' Soldier of the Rank of Private (Pseudo 'not real' Names) when asked onthe military potential risks that could increase sexual harassment among the interaction of soldiers in the Non-Commissioned Ranks individually and severally had this to say:

"Am happy to be asked once again on my view is that although it appears that there are no prevention measures I know how harassers behave and as such I avoid them ............ "

The next sub-paragraph discusses the similarities with respect to question two.

\section{Discussion of Research Findings to the Research Question Two (Similarities with other reviewed literature)}

There was sufficient evidence to show that the verbal and non-verbal sexual harassment that is experienced by junior female victim soldiers was as a result of bully perpetrator characteristics or behaviors that were exhibited by the senior male harassers. Additionally, there was also overwhelming evidence to show that female non-victim soldiers that initially said they understand sexual harassment had also seen or heard that bully characteristics or sexist behavior that were exhibited by the senior male harassers. This type of risk later led to the female junior soldiers being victims. 
There was also sufficient evidence to show that the verbal and non-verbal sexual harassment that is experienced by junior female victim soldiers was as a result of oppressive perpetrator characteristics or behaviors that were also exhibited by the senior male harassers. This type of risk also led to the female junior soldiers being victims. The non-victims soldiers that understand sexual harassment have also heard or seen the same events.

These findings are consistent with other studies. This is because there are studies that came up with similar findings in which bully or oppressive behavior as potential risks led to sexual harassment of the victims. This similarity was also seen from other studies at Global, African and Zambian perspectives.

For example when Rosen and Martin (1998) when examining childhood maltreatment as a risk factor for sexual harassment also established perpetrator bully and oppressive behaviors as leading to the problem in the United States of American Army.

Additionally, another study by Rosen and Martin (2000) whose aim was to examine personality characteristics that had the capacity of increasing sexual harassment also found that this would be through the risk of male discriminatory behavior against the females in the united states of American Army.

Another study that had similar findings to that of this study was conducted by Leskinen and group in 2011. According to Leskinen e'tal (2011) whose aim was to broaden the understanding with regard to gender harassment also found out that there was discrimination and oppressive behavior towards the females in the United States Army. This is believed to have led to non-verbal and verbal sexual harassment seen through non-sexual cooperation between the male and women.

The reason for the similarity in the findings with other studies regarding the perpetrator behaviors that lead to the risk of sexual harassment is largely because of similar environmental characteristics. These similar environmental internal characteristics may be seen through the differences in between the senior ranks and junior ranks which emphasize strict obedience and discpline. This researcher' perspective is also consistent with other studies. For exampleConformity, obedience, and the different hierarchical gender power relations between those in the lower ranks and higher ranks are a major factor that has contributed to the existence of sexual harassment in the military organizations (UNSC, 2008).

There was also sufficient evidence to show that the verbal and non-verbal harassment that was experienced by victims was usually not reported for fear of revenge from the senior soldiers. This individual risk was recorded from the victims. These findings by this study were also consistent with other reviewed literature.

For example Rosen and Martin (1998) when examining the childhood maltreatment history as a risk factor for sexual harassment in the American Army also found that the victims were not reporting the senior perpetrators to the relevant authorities about the problem.

Another study by the duet of Valerie and Cynthia (2016) aimed at understanding sexual harassment in the military by reviewing policy and trends in relation to why it is more pronounced than the civilians also that the victims were not reporting the cases to the relevant authorities.

Additionally Jana (2003) also found that the victims were not reporting the cases of being sexually harassed.

The reason for these similarities with other studies from the perspective of this study may be assumed to be as a result of fear of being punished through revenge by the perpetrator. This researcher perspective or view is also consistent with Jana' study in 2003 above.

There was also sufficient evidence to show that military risk factors such as lack of proper written and documented measures against sexual harassment resulted in the occurrence of the problem.This finding by this study was also consistent with other reviewed literature.

For example when Rosen and Martin (1998) when examining effects of the three types of unwanted sexual harassment experiences on the psychological wellbeing of soldiers also found that lack of written preventive measures from the organization led to the problem. 
Further another study by the duet of Valerie and Cynthia (2016) when reviewing the policy and trends in relation to how they increased sexual harassment also found that the United States Army did not have written preventive measures hence the problem.

Furthermore, another study by Harris et al (2017) also concluded that both individual and organizational climates and factors are important. However, organizational climate or context had less to do with culture or unit cohesion, but more to do with tolerance of sexism.

The research findings by Miller et al (1997) in the United States Army for this study showed that some military men believed that military women are the powerful gender. The research further recorded that unwelcome verbal and non-verbal actionwere experienced from bully female harassers.

Finally having discussed the findings in relation to the similarities with other studies it is also important to look at the dissimilarities.

\section{Discussion of Research Findings to the Research Question Two (Dissimilarities with other reviewed literature) (New Knowledge)}

There was sufficient combined individual evidence from both male and female victim/non-victim soldiers showing that Stalking Sexist/Perpetrator/Sexual Harassers as a potential risks factors increases sexual harassment among the interaction of soldiers.

The reason for the new knowledge is that, it is assumed that no other study has looked at both victims and non-victims showing empirical evidence of Stalking harassers as a potential risk factor that increases sexual harassment among the interaction of soldiers .

Therefore the study as indicated under the significance is important because it has given an insight into how stalking harassers as a potential risk factor increases of sexual harassment among the interaction of soldiers in selected military camps.

\section{Discussion of Research Findings to the Research Question Two (Theoretical Frameworks from reviewed literature)}

Based on the research findings of this study there was sufficient evidence to show that male discriminatory harassers did so because the female victim soldiers are in the minority.

Having been anchored on the theory of tokenism (1998) whose main thrust is that groups in the minority are more likely to be discriminated and marginalized by a group in the majority, it was established it was also in support of the findings.

These findings are also supported (Matakala 2015) on page 66 who also found that due to being in the minority female soldiers were likely to be sexually harassed. He also used the token theory.by Kamir (1998)

Furthermore, based on the research findings of this study there was sufficient evidence to show that male discriminatory harassers victimized the female victim soldiers in large camps. Because of the difference in the ranks and authority it was easy for the senior ranked males to harass the junior female soldiers.

The findings of the present study are supported by the theory of gender power relations by Michael Foucault (1975). This is because he postulated that the ability to have firm control and suppression of others through sexual harassment lies in the differential hierarchical rank structure based on who holds authority and power. The theory further states anyone who holds a high military rank in terms of authority and power has the capacity to control others in large military camps held by a senior field ranks.

The strength of this theory is that it also examined the large Hessian Military camps headed by senior field ranks which have the potential risk for sexual harassment occurrence.

\section{Discussion of Research Findings to the Research Question Two (Conceptual Framework of the study in relation to study findings)}

There was sufficient evidence to show that Discriminatory, Oppression and Inferiority Complex Harassers as perpetrator potential risk factors are responsible for sexual harassment among the 
interaction of soldiers. There was also sufficient evidence to show that Individual non-reporting as a potential risk factor was responsible for sexual harassment among the interaction of soldiers. Lastly, there was also sufficient evidence to show that lack of written preventive measures against sexual harassment as a military potential risk factor is responsible for sexual harassment among the interaction of soldiers.

These findings have as a result validated the assumption for the study Explanatory Conceptual Framework. This is with regard to main relationship between the independent and dependent variables respectively.

This is because the explanatory conceptual framework, assumed that the , measurement of Military organizations, Sexist/Perpetrators and Individual Potential Risk Factors as an (Independent Variable or Construct) may be responsible for Sexual Harassment which is the (Dependent Variable or Construct).

\section{Research Question Number Three (03).}

(3) What protective behaviors do Zambian soldiers adopt against the risk factors of being sexually harassed in selected military camps?

\section{Sub Thematic Questions:}

- Do you protect yourself against (low, medium or high) perceived or awareness levels of risk factors towards the possibility of being sexually harassed?

- In your own opinion why do you adopt or not adopt protective behavior against the (low, medium or high) awareness level towards the possibility of being sexually harassed in relation to (low, medium or high) severity or unpleasantness?

- What type of protective behaviors do you use against risk of sexual harassment?

\subsubsection{Research Findings to the Research Question Three (General summary of findings).}

(3) What protective behaviors do Zambian soldiers adopt against the risk factors of being sexually harassed in selected military camps?

The study combined responses from both the victim and non-victim participants. This was with regards to the adoption of protective behaviors by Zambian soldiers against the risk factors of being sexually harassed in selected military camps.

The study findings showed that both the male and female victim soldiers, did not adopt any protective behavior against the high perceived or awareness level towards possible risk factors of being sexually harassed.

A follow up question to this prevailing situation showed that the female victims feared to protect themselves against the low severity of being sexually harassed because they would end up with some form of revenge from the male perpetrators. Additionally, it was also established that the females lacked knowledge of the useful protective initiatives. Furthermore, the follow-up question to the male victims regarding why they do not adopt protective behavior's to the low severity of sexual harassment also showed that they lacked knowledge of the useful protective initiatives.

The study findings go ahead to show that both the male and female non-victim soldiers, adopted protective behavior against the high perceived or awareness levels towards the possible risk factors of being sexually harassed.

A follow up question to this prevailing situation showed that the female non-victim soldiers justification for adopting protective initiatives against the high severity of being sexually harassed was duo in nature. This was with regard to fear of affecting personal health and prior-knowledge of the perpetrator characteristics. The protective initiatives for the female non-victim soldiers included escape and evasion, avoidance of lone movement and stick friendship protective strategies respectively. This was done against the male perpetrator ranks of privates, lance corporals, corporals and sergeants that are senior in terms of promotion or a step ahead in rank. 
A similar follow up question to this prevailing situation showed that the male non-victims justification for adopting protective initiatives to the high severity or unpleasant situation was because of the fear that they would end up being regarded as weak men hence the justification. The protective initiatives for the male non-victim soldiers were the escape and evasion.

Sub-Theme Number One: Protective Behavioral Status against (high, medium or low) perceived or awareness towards the possible risk factors of being sexually harassed among the interaction of soldiers: Male and Female Victim Verbatims.

Protective behavioral status against the high, medium or low perceived or awareness towards the possible risk factors of being sexually harassed(Theme One).

According to an individual Female 'Victim' Soldier of the Rank of Private by the name of Josephine Daka, Josephine Mtonga , Josephine Phiri, Lance Corporal by the name of Pascalina Chilongoshi, Amina Abili, Amina Ability Corporal by the name of Jeannie Shapa, Sheba Babie, Janet Shapa Sergeant by the name of Joyce Kalebwe, Brenda Kaloshi and Joyce Mulemwa (Pseudo 'not real' Names) when asked onthe Protective behavioral status against the high, medium or low perceived or awareness towards the possible risk factors of being sexually harassed among the interaction of soldiers in the Non-Commissioned Ranks individually and severally had this to say:

"Having chatted with you at individual level last time ... Once again Iam very happy to be given another chance to yet again provide an answer to this question.............................my awareness towards the towards the possibility of risk factors for being sexually harassed is high.......... I have also been verbally and non-verbally harassed...... on my protective behavioral status.... I must say that I do not protect myself and I have a reason for not doing so..................iam not aware of any protective behavior that could be useful.....

Justification for Not Adopting or Adopting Protective Behaviour against the high perceived or awareness levels towards the possible risk factors of being sexually harassed despite its high, medium or low severity (Theme Two).

Fear of revenge as a justification for non-adoption of protective behavior against the high perceived or awareness levels towards the possible risk factors of being sexually harassed in relation to the medium severity (sub-theme)

Another Individual Female 'Victim', Soldier of the Rank of Private by the name of Josephine Phiri Lance Corporal by the name of Pascalina Chilongoshi, Amina Abili, Corporal by the name of Jeannie Shapa and Sheba Babie, (Pseudo 'not real' Names) ) when asked on theJustification For Not Adopting Protective Behaviour against the high perceived or awareness level towards the possible risk factors of being sexually harassed in relation to low, medium or high severity among the interaction of soldiers in the Non-Commissioned Ranks Individually and severally had this to say:

" ...................... wish to once again thank for allowing me to Providing an answer..................... when I reported a (senior male private, Lance corporal , Corporal) he started punishing me whenever I meet............................... Therefore, $l$ don't also protect myself because the severity of being sexually harassed is medium.

Lack of protective knowledge as a justification for non-adoption of protective behavior against the high perceived or awareness level to risk factors of being sexually harassed and medium severity (sub-theme)

Another individual Female 'Victim' Soldier of the Rank of Private by the name of Josephine Daka, Josephine Mtonga , Josephine Phiri, Lance Corporal by the name of Pascalina Chilongoshi, Amina Abili, Amina Ability Corporal by the name of Jeannie Shapa, Sheba Babie, Janet Shapa Sergeant by the name of Joyce Kalebwe, Brenda Kaloshi and Joyce Mulemwa (Pseudo 'not real' Names) when asked on theJustification For Not Adopting Protective Behaviour against the high perceived or awareness level towards the possible risk factors of being sexually harassed in relation to low, medium or high severity among the interaction of soldiers in the Non-Commissioned Ranks Individually and severally had this to say:

"The severity of sexual harassment is medium but I don't like being a victim whenever I think of reporting a (senior male private, Lance corporal, Corporal) I always fear to be 
punished....... l also don't know any protective behaviors' that could be used to avoid the risk factors of being sexually harassed........ after high possibility awareness which eventually happened..... ,

Protective behavioral status against the high, medium and low perceived or awareness to possible risk factors of being sexually harassed (Theme)

According to an individual Male 'Victim' Soldier of the Rank of Private by the name of Thomson Kunda, Rodrick Palula, Antony Phiri, Lance Corporal by the name of Alexander Msiska, Stanley Chipowe, Floyd Kamanga, Corporal by the name of George Muyeba, James Banda and Abraham Mubita (Pseudo 'not real' Names) when asked onthe Protective behavioral status against the high, medium and low perceived or awareness to possible risk factors of being sexually harassed among the interaction of soldiers in the Non-Commissioned Ranks individually and severally had this to say:

“.............my protective behavioral status against the high perceived risk for possibility of being sexually harassed is that I do not protect myself and I have a reason for not doing so...................iam not aware of any protective behavior that could be useful.

Justification for Not Adopting or Adopting Protective Behaviour against the high perceived or awareness levels to possible risk factors of being sexually harassed despite its high, low or very low severity (Theme Two).

Lack of protective knowledge as a justification for non-adoption of protective behavior against the high perceived or awareness level to risk factors of being sexually harassed and medium severity (sub-theme)

Another individual Male 'Victim' Soldier of the Rank of Private by the name of Thomson Kunda, Rodrick Palula, Antony Phiri, Lance Corporal by the name of Alexander Msiska, Stanley Chipowe, Floyd Kamanga, Corporal by the name of George Muyeba, James Banda and Abraham Mubita (Pseudo 'not real' Names) when asked on the Protective behavioral status against the high, medium and low perceived or awareness to possible risk factors of being sexually harassed among the interaction of soldiers in the Non-Commissioned Ranks individually and severally had this to say:

" .................... l do not know protective behaviors that could be used to avoid the risk of being sexually harassed even though my awareness to the possibility is high and I hate being a victim........ The severity is medium"

Protective behavioral status against the high, medium or low perceived or awareness towards the possible risk factors of being sexually harassed(Theme One).

According to an individual Female 'Non-Victim' Soldier of the Rank of Private by the name of Judy Musoko, Joyce Musenge, Tizzy Lemba, Lance Corporal by the name of Patricia Chosi, Chonya Amasi, Chama Abby Corporal by the name of Chisoni Musonda, Chola Stella, Chipo Stella, Sergeant by the name of Aggie Musonda, Chilila Stella and Chilekwa Stella (Pseudo 'not real' Names) when asked onthe Protective behavioral status against the high, medium or low perceived or awareness of possible risk factors of being sexually harassed among the interaction of soldiers in the Non-Commissioned Ranks individually and severally had this to say:

"Once again Iam very happy to be given another chance to yet again provide an answer towards this question.............................. with respect to my protective behavioral status against risk of being sexually harassed.........I must state that that I do protect myself and my awareness towards the possibility of sexual harassment is high

Justification for Adopting or Not Adopting Protective Behaviour against the high perceived or awareness levels to possible risk factors of being sexually harassed due its high, medium or low severity or unpleasantness (Theme Two).

Fear of affecting personal health as a justification for adoption of protective behavior against the high perceived or awareness level to risk factors of being sexually harassed due to its high severity or unpleasantness (sub-theme.1.)

Another Individual Female 'Non-Victim', Soldier of the Rank of Private by the name of Judy Musoko, Joyce Musenge, Tizzy Lemba, Lance Corporal by the name of Patricia Chosi, Chonya 
Amasi, Chama Abby Corporal by the name of Chisoni Musonda, Chola Stella, Chipo Stella, Sergeant by the name of Aggie Musonda, Chilila Stella and Chilekwa Stella (Pseudo 'not real' Names) when asked on justification for Adopting Protective Behaviour against the high perceived or awareness levels to possible risk factors of being sexually harassed due its high, medium or low severity or unpleasantnessamong the interaction of soldiers in the Non-Commissioned Ranks Individually and severally had this to say:

"...................... wish to once again thank you for this chance of providing an answer ......... I fear to suffer from hypertension (BP)........... I fear to suffer from severe headache that is why I protect myself from being sexually harassed and its high severity or unpleasantness ......................"

Prior-Knowledgeof perpetrator characteristics as a justification for adoption of protective behavior against the high perceived or awareness level to risk factors of being sexually harassed due to its high severity or unpleasantness (sub-theme.2.)

Another Individual Female 'Non-Victim', Soldier of the Rank of Private by the name of Judy Musoko, Joyce Musenge, Tizzy Lemba, Lance Corporal by the name of Patricia Chosi, Chonya Amasi, Chama Abby Corporal by the name of Chisoni Musonda, Chola Stella, Chipo Stella, Sergeant by the name of Aggie Musonda, Chilila Stella and Chilekwa Stella (Pseudo 'not real' Names) when asked on the justification for Adopting Protective Behaviour against the high perceived or awareness levels to possible risk factors of being sexually harassed due its high, medium or low severity or unpleasantness among the interaction of soldiers in the Non-Commissioned Ranks Individually and severally had this to say: “

$i$ do know that the senior male sexual harassers take advantage of the lack of proper written measures against sexual harassment........... I do also know that they have discriminatory behavior towards junior women soldiers because we are in the minority............I also know who they are and as such I prepare ways of protective initiatives beforehand because iam also aware the severity is high

\section{Protective behavioral initiative types used against the risk of being sexually harassed(Theme} Three).

According to an individual Female 'Non-Victim' Soldier of the Rank of Private by the name of Judy Musoko, Joyce Musenge, Tizzy Lemba, Lance Corporal by the name of Patricia Chosi, Chonya Amasi, Chama Abby Corporal by the name of Chisoni Musonda, Chola Stella, Chipo Stella, Sergeant by the name of Aggie Musonda, Chilila Stella and Chilekwa Stella (Pseudo 'not real' Names) when asked ontheprotective behavioral initiative types used against the risk of being sexually harassed among the interaction of soldiers in the Non-Commissioned Ranks individually and severally had this to say: “ since $i$ do know that the senior male sexual harassers take advantage of the lack of proper written measures against sexual harassment.......... I normally use the escape and evasion protective initiative.......................i use the avoidance of movement protective initiative.........................I use the stick friendship protective initiative towards the high perceived risk of being sexually harassed......

Protective behavioral status against the high, medium or low perceived or awareness towards the possible risk factors of being sexually harassed(Theme One).

According to an individual Male 'Non-Victim' Soldier of the Rank of Private by the name of James Muko, Joe Senge, Taiza Lembani, Lance Corporal by the name of Joseph Chisi, Chonya Mwenya, Chama Chanda, Corporal by the name of Chisoni Musonda, Chewe Musonda, Chola Stanley and Chola Chitalu (Pseudo 'not real' Names) when asked onthe Protective behavioral status against the high, medium or low perceived or awareness of possible risk factors of being sexually harassed among the interaction of soldiers in the Non-Commissioned Ranks individually and severally had this to say:

"Once again Iam very happy to be given another chance to yet again provide an answer towards this question............................. with respect to my protective behavioral status against my high perceived or awareness to the possible risk factors of being sexually harassed.........I must state that that I do protect myself... 
Justification for Adopting or Not Adopting Protective Behaviour against the high perceived or awareness levels to possible risk factors of being sexually harassed due its high, low or very low severity or unpleasantness (Theme Two).

Prior-Knowledge of perpetrator and health characteristics as a justification for adoption of protective behavior against the high perceived or awareness level to risk factors of being sexually harassed due to its high severity or unpleasantness (sub-theme.1.)

Another Individual Male 'Non-Victim', Soldier of the Rank of Private by the name of James Muko, Joe Senge, Taiza Lembani, Lance Corporal by the name of Joseph Chisi, Chonya Mwenya, Chama Chanda, Corporal by the name of Chisoni Musonda, Chewe Musonda, Chola Stanley and Chola Chitalu (Pseudo 'not real' Names) when asked on the justification for Adopting Protective Behaviour against the high perceived or awareness levels to possible risk factors of being sexually harassed due its high, medium or low severity or unpleasantness among the interaction of soldiers in the Non-Commissioned Ranks Individually and severally had this to say:

" $i$ do know that the senior female sexual harassers take advantage of the junior male soldiers because of their inferiority complex behaviour towards junior male

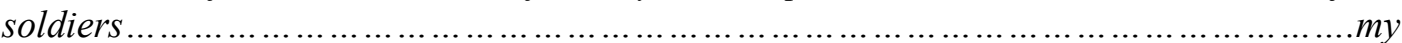

awareness towards the risk factors is high and iam aware that harassment can be of high severity to my health if I don't protect myself"'

\section{Actual Verbatims from (Male) Non-Victim Soldiers.}

\section{Protective behavioral initiative types used against the high perceived risk of being sexually} harassed (Theme Three).

According to an individual Male 'Non-Victim' Soldier of the Rank of Private by the name of James Muko, Joe Senge, Taiza Lembani, Lance Corporal by the name of Joseph Chisi, Chonya Mwenya, Chama Chanda, Corporal by the name of Chisoni Musonda, Chewe Musonda, Chola Stanley and Chola Chitalu (Pseudo 'not real' Names) when asked onthe Protective behavioral initiative types used against the high perceived risk of being sexually harassed among the interaction of soldiers in the Non-Commissioned Ranks individually and severally had this to say:

"I use the escape and evasion protective initiative towards the high perceived risk of being sexually harassed............................................. This is because I know the senior females that suffer from inferiority complex and how they behave towards junior male

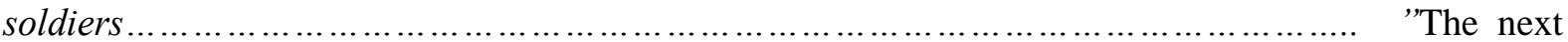
sub-paragraph discusses the above findings by contextualizing them with similar studies.

\section{Discussion of Research Findings to the Research Question Three (Similarities with other reviewed literature)}

There was sufficient evidence to show that the junior female non-victim soldiers feared to have their personal health affected if they did not adopt protective behaviors against severity of sexual harassment from the senior male sexual harassers. Additionally, there was also overwhelming evidence to show that female victim soldiers also feared that male soldiers would revenge hence the fear of adopting protective behaviors against the severe sexual harassment from senior male sexual harassers.

These findings are consistent with other studies. This is because there are studies that came up with similar findings in which their personal health affected when sexual harassment takes place. This similarity was also seen from other studies at Global, African and Zambian perspectives.

For example when Rosen and Martin (1998) when examining childhood maltreatment as a risk factor for sexual harassment also established perpetrator bully and oppressive behaviors as leading to the problem in the United States of American Army. This would later lead to chronic headache which is a personal health complication by the affected victims.

Additionally, another study by Rosen and Martin (2000) whose aim was to examine personality characteristics that had the capacity of increasing sexual harassment also found that this would be through the risk of male discriminatory behavior against the females in the united states of American 
Army. Some victims said they suffered hypertension which can also be regarded as a personal health complication, which they said was as a result of being sexually harassed.

According to Kim e'tal (2016) whose aim was to understand the influence of sexual harassment on mental health among female military personnel in the Korean Armed Forces, it was established that the victims had severe headache. This also was a personal health complication.

Another study that had similar findings to that of this study was conducted by Leskinen and group in 2011. According to Leskinen e'tal (2011) whose aim was to broaden the understanding with regard to gender harassment also found out that there was discrimination and oppressive behavior towards the females led to insomnia in the United States Army. This was also another personal health complication.

The reason for the similarity in the findings with other studies regarding the personal health complication that arise as a result of the risk of sexual harassment is largely because of similar environmental military characteristics. These similar environmental are also tied to the negative health effects of sexual harassment. This researcher' perspective is also consistent with other studies. For exampleThe health definition of sexual harassment considers, it as a 'Public health issue as it does not only affect the mental stability of an individual but it is also linked to other long term health problems that could put one at risk of high blood pressure, anxiety, depression and insomnia' (WHO, 2014). The study noted that work place sexual harassment did predispose victims to high risk of stroke and heart attacks as compared to those who did not experience the incidence (ibid,2014).

There was also overwhelming evidence to show that female victim soldiers also feared that male soldiers would revenge if they reported the senior male sexual harassers to the authorities. These findings by this study were also consistent with other reviewed literature.

For example Rosen and Martin (1998) when examining the childhood maltreatment history as a risk factor for sexual harassment in the American Army also found that the victims were not reporting the senior perpetrators to the relevant authorities about the problem because they feared that the repercussions would not be good.

Another study by the duet of Valerie and Cynthia (2016) aimed at understanding sexual harassment in the military by reviewing policy and trends in relation to why it is more pronounced than the civilians also that the victims were not reporting the cases to the relevant authorities they feared of being revenged.

Additionally Jana (2003) also found that the victims were not reporting the cases of being sexually harassed they feared a bad outcome once the perpetrator is called by the seniors.

The reason for these similarities with other studies from the perspective of this study may be assumed to be as a result of fear of being punished through revenge by the perpetrator. This researcher perspective or view is also consistent with Jana' study in 2003 above.

Finally having discussed the findings in relation to the similarities with other studies it is also important to look at the dissimilarities. Discussion of Research Findings to the Research Question Three (dissimilarities with other reviewed literature) (New Knowledge)

There was sufficient individual evidence from both male and female victim soldiers showing that they do not adopt protective behavioral initiative against high perceived or awareness possible risk factors of sexual harassment among the interaction of soldiers. This was largely because the severity was of being sexually harassed was medium and they lacked the knowledge of any protective initiatives.

There was also sufficient individual evidence from both male and female non- victim soldiers showing that they do adopt protective behavioral initiative against high perceived or awareness to possible risk factors of sexual harassment among the interaction of soldiers. This was largely because the severity of being sexually harassed was high and they had knowledge of the correct protective initiatives. The protective initiatives for the female non-victim soldiers included escape and evasion, avoidance of lone movement and stick friendship protective strategies respectively. This was done against the male perpetrator ranks of privates, lance corporals, corporals and sergeants that are senior in terms of promotion or a step ahead in rank. The protective initiatives for the male non- 
victim soldiers were the escape and evasion. This was done against the female perpetrator ranks of privates, lance corporals, corporals and sergeants that are senior in terms of promotion or a step ahead in rank.

The reason for the new knowledge is that, it is assumed that no other study has looked at both victims and non-victims regarding the situation on whether or not soldiers who perceive the risk of sexual harassment are aware of and/or adopt protective behaviours against sexual harassment.

Therefore, the study as indicated under the significance is important because it has given an insight into the different protective behaviors that Zambian Soldiers who perceive risk of sexual harassment in selected military camps utilize.

\section{Discussion of Research Findings to the Research Question Three (Theoretical Frameworks from reviewed literature)}

Based on the research findings of this study there was sufficient evidence to show that both male and female victim/non-victim soldiers had either protected themselves or not depending on the level of their perceived or awareness towards the risk factors of sexual harassment .

It was established that both male and female victim soldiers had high perceived or awareness of possible risk factors of being sexually harassed with low severity but never adopted any protective initiatives.

It was also established that both male and female non- victim soldiers that had high perceived or awareness of possible risk factors of being sexually harassed with high severity adopted protective initiatives.

The study was anchored on the Tripartite Perceived Risk Model(Ferrer e'tal, 2016) which gives an insight into the perceived risks of a health threat and peoples subjective judgements about whether/whether or not they should develop protective behaviours based on severity knowledge. Key for this study is that people with Low perceived risk; arenot likely to come up with protective behaviors. While those with High perceived risk; are likely to come up with protective behaviors.

It must be noted that the findings of male and female non- victim soldiers had high perceived risk of being sexually harassed but adopting protective initiatives, is supported by the Tripartite Perceived Risk Model. This is because key for the model is people with High perceived risk; are likely to come up with protective behaviors (Ferrer e'tal, 2016).

However, It must be noted that the findings of male and female victim soldiers had high perceived risk of being sexually harassed but did not adopt any protective initiatives, is not supported by the Tripartite Perceived Risk Model because it has made a deviation. This is because key for the model is that only people with Low perceived risk; arenot likely to come up with protective behaviors (Ferrer e'tal, 2016).

The Tripartite Perceived Risk Model which was designed to be used on health risks. However, in this case it was used for the first time on sexual harassment. This is because the model is relevant to sexual harassment as it falls within the social and health definition for which this model was developed for. Sexual harassment is a public health issue which is linked to other severe long term health problems that could put one at the risk of high blood pressure, anxiety, depression and insomnia (WHO, 2014).

It is against this background that having deviated from the initial model there will be no modification but generation of a new theory. This is because sexual harassment is a new phenomenon on perceived risk concept. This assertion is supported by the fact in science, research is one of the main process by which data are collected to support, reject or modify theory or to develop new ones. Additionally a theory is an interpretation of phenomenon (Parahoo, 1997)

Furthermore since the model, looked at health risks that was tested on cancer patients I feel I have made a significant contribution because I used it for the first time on sexual harassment which is also considered as a public health condition (WHO, 2014).

I therefore feel my new way of explain phenomenon should be ascribed to my name. 
This is because inorder to draw such conclusions I have clarified academic arguments, and given reasons for this final documentation of my thinking (Mouton, 2016 and Moody 1990).

Therefore I wish to ascribe my new findings to myself as shown below:

Matakala et al 2021: Theory of Non-Victim and Victim High perceived risk of being sexually harassed and emerging protective behaviors among Zambian Soldiers;

The new theory is also ascribed to university of Zambia dons namely Dr Anne Namakando-Phiri a retired army colonel and professor Mubiana Macwang'i.

\section{Matakala (2021)'s Theory Of Non-Victim/Victim High Perceived Risk Perception of Sexual Harassment among Zambian Soldiers in Relation To Motivation of Protective Behaviours.}

Key for this theory is that the sub-population of both maleand female victim soldiers that had an overall high perceived risk of being sexually harassed, calculated from their high perceived or awareness of possibility with a medium severity risk never adopted any protective initiatives.

Individual available evidence from both male and female victim soldiers, showed that they do not adopt protective behavioral initiative against high perceivedrisk factorsof sexual harassment because of the fear of revenge and the general lack of knowledge about protective initiatives.

Key for this theory also indicate that the sub-population of both male and female non- victim soldiers that had an overall high perceived risk of being sexually harassed, calculated from their high perceived or awareness of possibility with a high severity in mind adopted protective initiatives.

Individual available evidence from both male and female non- victim soldiers showed that they adopt protective behavioral initiative against high perceived risk factors of sexual harassment among the interaction of soldiers. This was largely because they have knowledge of the correct protective initiatives against the high severity of sexual harassment. This knowledge arose from the fact that they feared to be affected with health risk such as insomnia and heart attacks if they are harassed. The protective initiatives for the female non-victim soldiers therefore, included escape and evasion, avoidance of lone movement and stick friendship protective strategies respectively. This was done against the male perpetrator ranks of privates, lance corporals, corporals and sergeants that are senior in terms of promotion or a step ahead in rank. The protective initiatives for the male non-victim soldiers were the escape and evasion. This was done against the female perpetrator ranks of privates, lance corporals, corporals and sergeants that are senior in terms of promotion or a step ahead in rank.

The new theory deviated from existing theory which state that only people with low perceived risks are likely to protect themselves from a health threat (Ferrer et al 2016). The new theory comes up with a new explanation that victim soldiers with high perceived risk did not protect themselves with an academic support by (Moody 1990).

The new theory confirms and agrees with existing theory that people with high perceived risks are likely to protect themselves from a health threat (Ferrer et al 2016), However, the new explanation is based on a new phenomenon sexual harassment which is also a public health (WHO, 2014).

In line with risk analysis, a high risk of sexual harassment will always demand top priority in terms of adopting protective behaviors. A risk has two facets which are possibility and severity characteristics. Both non-victims and victims of sexual harassment had a high perceived risk. This was calculated through the $3 * 3$ risk matrix. The matrix shows that any colour in red represents a high risk of sexual harassment hence top priority when it comes to protection. The product of a risk= possibility multiply by severity.

Horizontal Axis=begins with 1. low, 2.medium and 3.high increasing to the right which is the severity level for being sexually harassed

Vertical Axis = begins with 1. low, 2.medium and 3. high increasing to the upwards which represents possibility level of being sexually harassed.

The Non-Victim recorded high possibility (3) multiplied by high severity (3) of being sexually harassed $=$ overall risk $(9)$. 
The Victims recorded high possibility (3) multiplied by medium severity (2) of being sexually harassed=overall risk (6).

Both overall risks that is 6 and 9 are represented by the red colour hence high risk which demand top priority as shown hereunder.

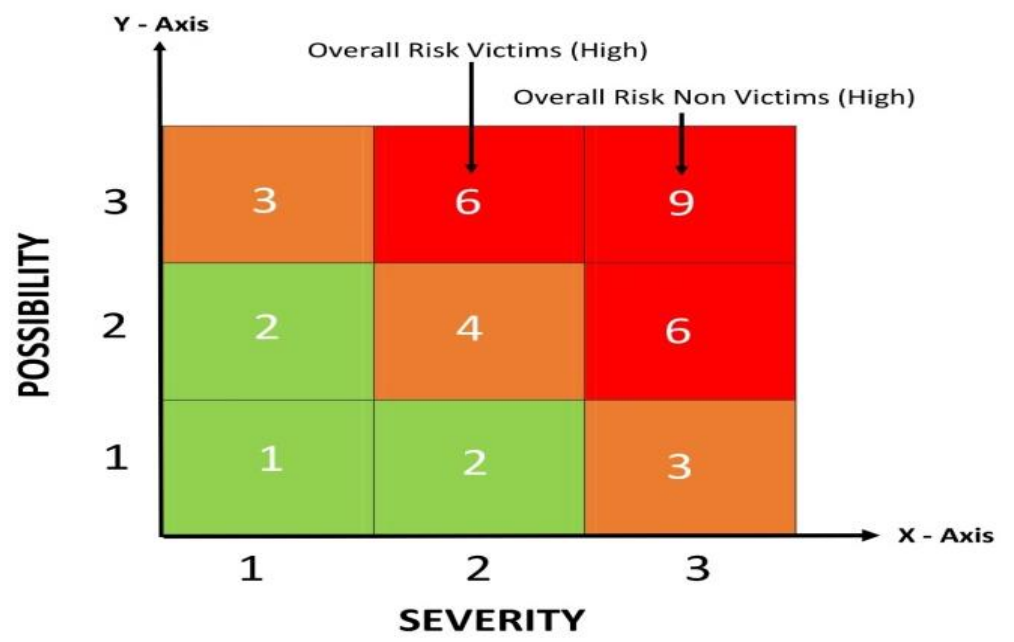

Source: Field Data

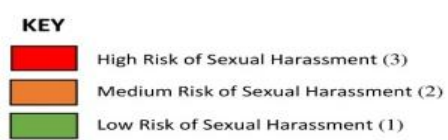

Figure1. 3*3 Risk Analysis Matrix showing risk status for both non-victims and victims of sexual harassment in relation to the generated theory (Matakala 2021)

Source: Field Data

KEY/LEGEND

$\begin{array}{lll}\text { Red }=\text { High } & {[3]} & \text { (risk of sexual harassment) } \\ \text { Orange=Medium } & {[2]} & \text { (risk of sexual harassment) } \\ \text { Green= Low } & {[1]} & \text { (risk of sexual harassment) }\end{array}$

\section{Research Question Number Four (04)}

4) How do perceived risks of being sexually harassed motivate protective behaviors among Zambian soldiers in selected military camps?

\section{Thematic Sub Questions:}

- In your own opinion how does (non-victim high perceived risk) of sexual harassment motivate protective behaviors?

- How effective are the adopted protective behaviors do you use against risk of sexual harassment?

3.2.4. Research Findings to the Research Question Four (General summary of findings).

(4) How do perceived risks of being sexually harassed motivate protective behaviors among Zambian soldiers in selected military camps?

The study looked at responses non-victim participants. This was with regards to how do perceived risks of being sexually harassed motivate protective behaviors among Zambian soldiers in selected military camps.

The study findings showed that both the male and female non-victim soldiers with high perceived risk of being sexually harassed were motivated to adopted protective behaviors.

Male Bully/Discriminatory perpetrator awareness motivated the adoption of female non-victim protective initiatives. The protective initiatives for the female non-victim soldiers included escape and evasion, avoidance of lone movement and stick friendship protective strategies respectively. It was established that the non-victim females did so through the principle of see without being seen which gave rise to the escape and evasion protective initiative. It was also established since they knew the 
characteristics of the senior male sexual harassers they avoided lone movements. Which was a protective initiative when moving in areas where they are found? It was also established that they moved in a group of four when approaching such people as a protective initiative.

This was done against the male perpetrator ranks of privates, lance corporals, corporals and sergeants that are senior in terms of promotion or a step ahead in rank.

The study findings further showed that Female Bully/Inferiority Complex perpetrator awareness motivated the adoption of male non-victim protective initiatives. The protective initiatives for the male non-victim soldiers was the escape and evasion. It was established that male non-victim soldiers that had perceived high risk female inferiority complex awareness of being sexually harassed were motivated to take up protective initiatives. It was established that the male non-victims did so through the principle of see without being seen which gave rise to the escape and evasion protective initiative. This was done against the female perpetrator ranks of privates, lance corporals, corporals and sergeants that are senior in terms of promotion or a step ahead in rank.

(Non-victim high perceived risk) of sexual harassment and how it motivates protective behavior: Female Verbatims. (Theme Number One)

The Female Escape And Evasion Protective Initiative adopted by Non-Victims as a Motivation towards the high perceived risk of being sexually harassed based on senior Male Bully/Discriminatory characteristic identification (Sub-theme one).

According to an individual Female 'Non-Victim' Soldier of the Rank of Private by the name of Judy Musoko, Joyce Musenge, Tizzy Lemba, Lance Corporal by the name of Patricia Chosi, Chonya Amasi, Chama Abby, Corporal by the name of Chisoni Musonda, Chola Stella, Chipo Stella Sergeant by the name of Aggie Musonda, Chilila Stella and Chilekwa Stella (Pseudo 'not real' Names) when asked on how the non-victim high perceived risks of being sexually harassed motivate protective behaviors among the interaction of soldiers in the Non-Commissioned Ranks individually and severally had this to say:

“.........At training I was taught to see the enemy first without them seeing you because it meant I would eliminate him................. since I know that senior male perpetrators are discriminatory.................... senior male sexual harassers are bullies I use the principle of see without being seen which helps me to do the escape and evasion protective initiative..... that is why it is difficult for me to be sexually harassed...

The Female Avoidance Of Lone Movement Protective Initiative adopted by Non-Victims as a Motivation towards the high perceived risk of being sexually harassed based on senior Male Bully/Discriminatory characteristic identification (Sub-theme two).

Another individual Female 'Non-Victim' Soldier of the Rank of Private by the name of Judy Musoko, Joyce Musenge, Tizzy Lemba, Lance Corporal by the name of Patricia Chosi, Chonya Amasi, Chama Abby, Corporal by the name of Chisoni Musonda, Chola Stella, Chipo Stella Sergeant by the name of Aggie Musonda, Chilila Stella and Chilekwa Stella (Pseudo 'not real' Names) when asked on how the non-victim high perceived risks of being sexually harassed motivate protective behaviors among the interaction of soldiers in the Non-Commissioned Ranks individually and severally had this to say:

“.........As I have already said I know the senior male soldiers that are not only bullies but also like using discriminatory language..... because of this when I am near them I avoid being with them when I am alone.... that is why it is difficult for me to be sexually harassed......

The Female Stick Friendship Protective Initiative adopted by Non-Victims as a Motivation towards the high perceived risk of being sexually harassed based on senior Male Bully/Discriminatory characteristic identification (Sub-theme three).

Another individual Female 'Non-Victim' Soldier of the Rank of Private by the name of Judy Musoko, Joyce Musenge, Tizzy Lemba, Lance Corporal by the name of Patricia Chosi, Chonya Amasi, Chama Abby, Corporal by the name of Chisoni Musonda, Chola Stella, Chipo Stella Sergeant by the name of Aggie Musonda, Chilila Stella and Chilekwa Stella (Pseudo 'not real' Names) when asked on how the non-victim high perceived risks of being sexually harassed motivate 
protective behaviors among the interaction of soldiers in the Non-Commissioned Ranks individually and severally had this to say:

“...... prior knowledge of the characteristics of the male sexual harassers it be bully or discriminatory has made me to going near these people when I am in a group of four which is also called a sick...........I have noticed that they don't do anything ........that is why it is difficult for me to be sexually harassed...

The next category looks at the male non-victims.

Actual Verbatims from (Male) Non-Victim Soldiers.

(Non-victim high perceived risk) of sexual harassment and how it motivates protective behavior: Male Verbatims. (Theme Number One)

The Male Escape And Evasion Protective Initiative adopted by Non-Victims as a Motivation towards the high perceived risk of being sexually harassed based on senior Female Bully/Discriminatory characteristic identification (Sub-theme one).

According to an individual Male 'Non-Victim' Soldier of the Rank of Private by the name of James Muko, Joe Senge, Taiza Lemba, Lance Corporal by the name of Joseph Chisi, Chonya Mwenya, Chama Chanda, Corporal by the name of Chewe Musonda, Chola Stanley and Chola Chitalu (Pseudo 'not real' Names) when asked on how the non-victim high perceived risks of being sexually harassed motivate protective behaviors among the interaction of soldiers in the Non-Commissioned Ranks individually and severally had this to say:

".........senior female sexual harassers have inferiority complex and I know them I use the principle of seeng without being seen which helps me to do the escape and evasion protective initiative.....

The next sub-paragraph discusses the findings in relation to similar studies.

\section{Discussion Of Research Findings To The Research Question Four}

\section{Similarities Of Research Findings With Reviewed Literature}

There was no sufficient evidence to show similar findings from the reviewed literature.

The findings of the present study are therefore, not consistent with other studies. This is because it appears there are no studies that have raised questions as a research gap on whether or not soldiers who perceive the risk of sexual harassment are aware of and/or adopt protective behaviours against sexual harassment.

Finally having discussed the findings in relation to the similarities with other studies it is also important to look at the dissimilarities.

\section{Dissimilarities/ Lessons Learnt from the Research Findingsof the Research Question Four (New Knowledge)}

There was sufficient individual evidence to show that female non-victim soldiers that had perceived high risk male bully/discriminatory awareness of being sexually harassed were motivated to take up protective initiatives. It was established that they did so through the principle of see without being seen which gave rise to the escape and evasion protective initiative. It was also established since they knew the characteristics of the senior male sexual harassers they avoided lone movements which was a protective initiative when moving in areas where they are found. It was also established that they moved in a group of four when approaching such people as a protective initiative.

There was also sufficient individual evidence to show that male non-victim soldiers that had perceived high risk female inferiority complex awareness of being sexually harassed were motivated to take up protective initiatives. It was established that they did so through the principle of see without being seen which gave rise to the escape and evasion protective initiative.

The reason for the new knowledge is that, it is assumed that no other study has looked at both female and male non-victims regarding the situation on how non-victim high perceived or awareness of possible risks of being sexually harassed motivate protective behaviors among Zambian soldiers in selected military camps. 
Therefore the study as indicated under the significance is important because it has given an insight into the different protective behaviors that Zambian Soldiers who perceive risk of sexual harassment in selected military camps utilize.

\section{Theoretical Frameworks and Lessons Learnt from the Research Findingsof the Research Question Four}

Based on the research findings of this study there was sufficient evidence to show that both male and female non-victim soldiers that had perceived high risk male bully/discriminatory awareness of being sexually harassed were motivated to take up protective initiatives. It was established that they did so through the principle of see without being seen which gave rise to the escape and evasion protective initiative. It was also established since they knew the characteristics of the senior male sexual harassers they avoided lone movements. Which was a protective initiative Iwhen moving in areas where they are found? It was also established that they moved in a group of four when approaching such people as a protective initiative.

There was also sufficient individual evidence to show that male non-victim soldiers that had perceived high risk female inferiority complex awareness of being sexually harassed were motivated to take up protective initiatives. It was established that they did so through the principle of see without being seen which gave rise to the escape and evasion protective initiative.

Each of the interviewed individual non-victims felt that each time they adopted the protective initiatives among their interactions they do lnot become victims of verbal and non-verbal sexual harassment. It is against this background that they felt this was what was workable and that it made great sense. This is because the protective initiatives are helpful.

These findings are supported by the ethno-methodology paradigm.

This is because the Ethno methodology paradigms are also interested in what makes sense for individuals or groups of people in a given community and the methods they use to cope or protect themselves (Garfinkel, 1967). It was popularized by Harold Garfinkel.

\section{RESEARCH ORIGINALITY}

This chapter is in two parts

Part A is the general presentation on the originality of the Thesis. Part B is the presentation of the non-victim protective initiatives against the high perceived risk of being sexually harassed and how they were practically validated by victims as a test of effectiveness. The chapter closes with a summary.

\subsection{General Research Originality of the Thesis}

The concept of originality in the doctoral research tries to outline ways in which the contribution towards academic knowledge base can be demonstrated. It is the perspective of the Doctor of Philosophy candidate. Further, in this thesis the originality is marked as the final embodiment of the research project based on the documentation of the researcher's thinking. It is a statement that is accompanied by the act of advancing and clarifying arguments, reasons and evidence for reaching certain conclusions based on the principles of logic of validation in every research thesis. Depending on the faculty or school of thought the statement of research originality is situated as a separate chapter after the discussion of findings or after the conclusion of the study. This because the key findings to the research questions of the study, will have been achieved at this stage, enough to draw conclusions as well as coming up with specific validated recommendations. This study therefore, situates the statement of research originality after the discussion of findings as a separate chapter before the conclusion chapter. It is important that $\mathrm{i}$ address the issue of research originality in this thesis, not only because it is some form of criteria for assessing quality in Doctoral Research, but also because it ensures that the study made significant contribution to the body of knowledge. It is therefore, important for this thesis to demonstrate critically how and in what ways the significant contribution to the body of knowledge was achieved. The dual significant contribution to the body of knowledge was based on the identified research gap. This is because it appeared no other study tried to understand the protective behaviours that soldiers who perceive risk of being sexually harassed 
adapt with respect to two sub-population groups Victims and Non-Victims. The researcher went on to produce new knowledge based on his own perspective using the existing ideas of other studies to back up his argument and clarified these with reasons in relation with what other studies had said on sexual harassment before using the resultant evidence for drawing of conclusions being stated as well as leading to a proposal of recommendations signified by the logic validation. (Creswell, 2009). Let me now demonstrate my research originality as what also constitutes what my thesis is all about through 'what we did not know that we now know' in relation to the initial identified research gap. The research gap was based on the 'understanding of both the non-victim and victim protective behaviours in relation to their perceived risk for sexual harassment among Zambian soldiers'. The research established that victim soldiers that had knowledge about the high perceived risk factors of being sexually harassed did not protect themselves because they lacked the knowledge of how to do so. However, it was also established that non-victim soldiers that had knowledge about the high perceived risk factors of being sexually harassed did protect themselves in three ways that are shown hereunder;

1. There was sufficient evidence to show that female non-victims that exhibited a high perceived risk of being sexually harassed adopted three ideal protective initiatives. These female non-victim protective initiatives among others included escape and evasion, avoidance of lone movement and stick friendship protective strategies respectively. It was established that they did so through the principle of see without being seen which gave rise to the escape and evasion protective initiative. It was also established since they knew the characteristics of the senior male sexual harassers they avoided lone movements. Which was a protective initative when moving in areas where they are found? It was also established that they moved in a group of four when approaching such people as a protective initiative.

This was to be done against the male perpetrator ranks of privates, lance corporals, corporals and sergeants that are senior in terms of promotion or a step ahead in rank in case of a suspected threat.

2. There was also sufficient evidence to show that male non-victims that exhibited a high perceived risk of being sexually harassed adopted three ideal protective initiatives have since established one ideal protective behavior. These included escape and evasion protective strategy. It was established that they did so through the principle of see without being seen which gave rise to the escape and evasion protective initiative.

This was done against the female perpetrator ranks of privates, lance corporals, corporals and sergeants that are senior in terms of promotion or a step ahead in rank in case of a suspected physical threat.

Equipped with the aforementioned non-victim protective initiatives as what we did not know that is now known I assumed that the protective initiatives may be of help to both victims and nonvictims of sexual harassment subject to logic research validation.

The effectiveness of the resultant non-victim protective initiatives as my proposed recommendations against the risk of sexual harassment among soldiers in Zambia was later subjected to a logic research validation test.

In order to test the effectiveness i purposefully selected few victim soldiers that had knowledge about the high perceived risk factors of being sexually harassed but did not protect themselves because they lacked the knowledge of how to do so.

I then proceeded to brief victim soldiers about how I felt they could protect themselves based on what I had established from the non-victim perspectives.

At the end of each individual victim briefing I asked if they had any questions before asking them to go and practice what they had heard for a period of four weeks. I told them that after this briefing I would call for another interview. When the non-victims reported back they testified that they never suffered sexual harassment again.

With this demonstration of my research originality I therefore, I had made significant contribution to the body of knowledge

The demonstration was in two phases shown hereunder: 
4.2. Effectiveness (Test) of The Non-Victim Protective initiatives against the high perceived risk factors of being Sexually Harassed by Perpetrators among Zambian Soldiers: A Zambian Perspective Guide.

The Non-Victim Protective initiatives against the high perceived risk of being Sexually Harassed by Perpetrators among Zambian Soldierswere subjected to an effectiveness test that was divided into two parts.

\section{First part}

Inclusion criteria for the victims of sexual harassment used in the practical- validation of the Non-victim Protective Initiatives/Preliminary instructions

The first part was based on the information provided by the researcher to the few selected individual female and male victims that participated in the study. The selected participants represented the various affected ranks which are privates, lance corporals, corporals and sergeants for the females. The male affected ranks included ranks of private, lance corporal and corporal. This was with specific reference to research question number three. The question looked at the protective behaviors Zambian soldiers adopt against the risk factors of being sexually harassed in selected military camps .This category was chosen because it was established that despite their high perceived risk of being sexually harassed they never adopted any protective initiatives. It was established that the reason for not adopting protective initiatives is because the lacked knowledge of the correct protective initiative. It is against this background that they were excluded from the forth research question. Only nonvictims were included. It is from the non-victims that the proposed recommendations have arisen from.

Therefore the female and male victim individuals were admonished to go and experiment from the proposed protective initiative recommendations based on the non-victim perspectives. This was to ascertain the workability of the proposed recommendations. The victims were told by the researcher that since they knew the perpetrator characteristics it would be in order to establish the effectiveness of the proposed recommendations. The selected victims were admonished to use the under mentioned protective initiatives or strategies;

1) Use of the male/female escape and evasion protective initiative against the perpetrator sexual harassers: Once the high perceived risk bully, discriminatory characteristics as well as the actual perpetrators are identified it becomes easy to use the protective initiative. This is because it will be easy to use the principle of 'see without being seen.' This means when the male or sexual harasser is seen first it becomes easy to do the escape and evasion protective initiative.

2) Use of the female stick friendship protective initiative for use against the senior male perpetrators of sexual harassment: Once the high perceived risk bully, discriminatory characteristics as well as the actual perpetrators are identified it becomes easy to use the protective initiative. Always moving in a group of four or stick friendship initiative makes it difficult for the senior male sexual harassers to strike.

3) Use of the female avoidance of lone movement protective initiative against the senior male perpetrators of sexual harassment: Once the high perceived risk bully, discriminatory characteristics as well as the actual perpetrators are identified it becomes easy to use the protective initiative. Always not moving alone in the presence of the known perpetrators based on characteristics they possess makes it difficult for the senior male harassers to have the opportunity of striking.

\section{Second Part}

Revelations From Individual Female And Male Victim Soldiers That Used The Proposed 'Formal Proactive Protective Initiatives Against The High perceived risk factors of Sexual Harassment': A Test/Validation For Effectiveness.

QUESTION: In your own opinion do you think the use of the proposed protective initiatives against the risk of being sexually harassed in the last four weeks of trials were effective? 


\section{Actual Individual Verbatims for the female victim soldiers.}

According to a Female Victim soldier of the Rank of Private by the name of Josephine Mtonga, Josephine Phiri, Josephine Daka, Lance Corporal by the name of Pascalina Chilongoshi, Amina Abili, Amina Ability, Corporal by the name of Jeannie Shapa, Sheba Babie, Janet Shapa Sergeant by the name of Joyce Kalebwe, Joyce Mulemwa and Joyce Mulemwa (Pseudo 'not real' Names) when asked on the effectiveness of the proposed formal proactive protective initiatives against the high perceived risk of sexual harassment among the interaction of soldiers in the Non-Commissioned Ranks Individually and severally said that:

"............ knowledge of perpetrator characteristics as well as the existing military culture that is characterized with obedience and discipline gives rise to a high perceived risk perception in me.................last time I said I can't protect myself because I lack the knowledge of any protective initiatives .........for fear of being Indiscipline due to the military culture........... but since you advised on what to do the last four weeks have not made me to be sexually harassed................. this is because I used the stick friendship protective initiative were I was in the company of four...................................................this because $I$ avoided moving alone.........................this is because I used the escape and evade protective behavior were I should be the first to see the enemy or the sexual harasser...

\section{Actual Individual Verbatims for the male victim soldiers}

According, to a Male Victim Soldier of the Rank of Private by the name of Thomson Kunda, Private Rodrick Palula, Antony Phiri, Lance Corporal by the name of Alexander Msiska, Stanley Chipowe, Floyd Kamanga, Corporal Abraham Mubita, George Muyeba and James Banda (Pseudo 'not real' Names) when asked on the effectiveness of the proposed formal proactive protective initiatives against high perceived risk of sexual harassment among the interaction of soldiers in the Non-Commissioned Ranks Individually and severally said that:

"Once again I thank you for the opportunity .... My contribution is that knowledge of perpetrator characteristics as well as the existing military culture that is characterized with obedience and discipline gives rise to a high perceived risk perception in me.................last time I said I could not protect myself......... for fear of being Indiscipline due to the existing military culture as well as lack of the knowledge of the correct protective initiatives ............ however, since you advised on what to do the last four weeks have not made me to be sexually harassed................. this is because I used because I used the escape and evade protective behavior were I should be the first to see the enemy..............."

In closing the session, the researcher thanked all the individual male and female victims or their valuable information and contributions.

As a result of the aforementioned views this thesis makes a considerable contribution to the body of knowledge and to some extent literature review which is scant in military studies that have looked at sexual harassment.

The conclusions drawn by this thesis are not imposed on the study, but reflect the experiences for both non-victims and victims for sexual harassment among the interactions of soldiers in selected military camps from the non-commissioned ranks. Therefore, this thesis has also contributed to the awakening debate by other countries including the Zambian Government on how to prevent sexual harassment not only in the military communities but also the countries as a whole. In Zambia this was done through the enactment of the SI number 15 of 2005, section 137A that criminalizes sexual harassment as well as coming up with gender desks for sensitization of in various headquarters of ministries. These also included the Ministry of Defense (MOD) and its security wings together with the Army.

To the best of the my knowledge some of the information and data gathered by this thesis have not been documented anywhere hence it can be said to be new knowledge. The justification lies in the fact that the answers to this thesis have been contextualized with other

The new knowledge on the formal adopted proactive recommendations against sexual harassment based on the perspective of the non-victims is the first of its kind among Zambian soldiers.

The next chapter is going to look at the conclusion, recommendations and suggestions for future research. 


\section{CONClusion And Suggestions For Future RESEARCH}

\subsection{Conclusion}

This thesis is important because it bridges the gap in knowledge with other studies that only included victims in sexual harassment investigations among soldiers. It was therefore, important to explore and understand, the link of Perceived Risk of sexual harassment and protective behaviours adopted by both the victim and non-victim soldiers if a suitable interventions had to be proposed for the military camps, as it is an under explored area. Four major themes were explored inorder to draw study conclusions as shown hereunder;

(1) Situation on perceived risk of being sexually harassed among Zambian soldiers.

The findings on the perceived risk perceptions of being sexually harassed revealed a high magnitude for both verbal and non-verbal actions. Only ranks between private and corporal continued being affected while the ranks above sergeant are also said to have experienced these actions within the same category. This situation gave rise to a high perceived or awareness towards the risk factors of being sexually harassed among the current affected category victim and non-victim soldiers.

(2) Risk factors associated with sexual harassment among Zambian soldiers.

The findings on the risk factors associated with sexual harassment were measured as (i) perpetrator (ii) individual weakness and (iii) military characteristics respectively. This was also done from the perspective of both victims and non-victim soldiers. Male/Female bully and discriminatory was among the recorded perpetrator characteristics. Furthermore, non-reporting of the perpetrators was recorded as an individual victim weakness which was not the case for the non-victims. Lack of written sexual harassment mitigation measures was recorded as a military characteristic that was also a risk factor for the prevalence of sexual harassment.

(3) Protective behaviors Zambian soldiers adopt against the risk factors of being sexually harassed.

The new findings on protective behaviors against the risk factors of sexual harassment deviated from existing theory. It was established victims never adopted protective behaviors fearing revenge, but non-victims protected themselves as they feared getting affected health-wise. In both cases the new explanation of phenomenon was a motivation against high perceived or awareness towards the risk factors of sexual harassment, hence Matakala's theory and others.

(4) Perceived risks of being sexually harassed and how they motivate protective behaviors among Zambian soldiers.

The findings from the non-victim on how the high perceived or awareness towards riskof being sexually harassed motivates protective behaviors revealed three protective initiatives. These were escape and evasion, stick and avoidance of lone movement protective initiatives. The escape and evasion was based on the principle of being able to see the known perpetrator first so that it is easy to avoid them. The avoidance of lone movement principle was meant not to come in the presence of the known perpetrator while alone. Lastly, the stick principle was made to ensure movement to the known perpetrator was done in a group of four making it difficult for harassment to take place.

Lastly, the resultant non-victim protective initiatives were later practically-validated by the victim soldiers as they also knew the bully/discriminatory perpetrator characteristics. The non-victim protective initiatives also worked effectively for the victims as shown from their testimonies.

\subsection{Suggestions for Future Research Studies}

Despite that the findings of this study can be extrapolated to the commissioned ranks, it is recommended that the next research study should include the said category. This should also be with respect to the concept of perceived risk and protective behaviors. This is because the present study has acted as a stepping stone for further research.

\section{REFERENCES}

[1] Allen, E. (2013) Worker Sexually Harassed by Manager in England, Mail Online News, London. 
Perceived Risk of Sexual Harassment and Protective Behaviours among Zambian Soldiers in Selected Military Camps

[2] Antecol H and Cobb-Clark D (2001) Men, Women and sexual harassment in the United States Army, Gender Issues Journal Volume 19, Issue (3), Pages 3-18.

[3] Backhouse, C. (2000) the Secret Oppression: Sexual Harassment of Working Women Manitoba, Macmillan Publishers, Manitoba.

[4] Baker, D. D, Jepestra, D. and Cutler, S. (1990). Perceptions of sexual harassment: A survey United States Military, Journal of psychology, 126 p 416-426

[5] Bell, E.M; Christina, M.D; Stephanie, A.V and Amy, E.S (2018) Victims of sexual harassment in the military: Understanding perceived risks and promoting recovery. Journal of Military Psychology Volume 30, issue no. 3, pp. 65-78.

[6] Bless, C. and Achola, P. (2001). Fundamentals of Social Research Methods, Pauline Publishers, Nairobi.

[7] Brandsaeter, J. and Widebery, S. (1992) Sexual Harassment and Social Behaviour, Sage Publications, London.

[8] Buchanan N T, Settles I H, Hall A T and O'Connor R C (2014) A review organisational strategies for reducing sexual harassment: Insights from the United States Army, Social Issues Journal, Volume 70, Issue (4) Pages 687-702.

[9] Butler, J. S. and Schimdike, J. M (2010) Theoretical traditions and modeling of sexual harassment within organizations: The military as data. Armed forces and society journal, 36, p193-222.

[10] Carey, M (2012), Qualitative research skills for social work: Theory and practice, Ashgate publishers, New York.

[11] Cameron, E. (2013) The New Labour Relations Act, South Africa, Juta and Company, Johannesburg.

[12] Charles, V. W; Gillian, F and Colin, T (2009).Sexual Harassment of women in the South African Navy, South African Journal of Psychology.

[13] Clavel, J. (1983) the Art of War by Sun Tzu, Random House Publishers, New York.

[14] Cooper, H. (2014). Pentagon Study Finds 50\% Increase in Reports of Military Sexual Harassments and Assaults. New Penguin Publishers, New York.

[15] Creswell, J.W. (2007). Qualitative Inquiry and Research Designs: Choosing Among Five Approaches, $2^{\text {nd }}$ ed. Sage Publishers, London.

[16] Creswell, J.W. (2009). Research design: Qualitative, Quantities and Mixed methods Approaches, Sage Publishers, New Delhi.

[17] Cohen, A. (2001). Review of Literature: Responses to "Empirical and Hermeneutic Approaches to Phenomenological research in Psychology, a Comparison" Gestalt, 5 (2) retrieved from http:www.ggej.org/5-2/reviewlit.html.

[18] Davidson, A. (2013). Military Sexual Harassment and Assault: Shameful Isn't Enough, The New Yorker Close Read Biog, New York.

[19] Deschamps, C. C. And Mills, A.F (2015) External Review into Sexual Harassment in the Canadian Armed Forces, Journal of Social issues p687-702.

[20] Davidson, A. (2013). Military Sexual Harassment and Assault: Shameful Isn't it Enough, The New Close Read Biog, New York.

[21] Egaden, C.L. (2012). Participatory Action/Toolkit Procedure using Future Search Research: A Modern Thought for Doctoral and Post-Doctoral Students, Sage Publications, London.

[22] EEOC (2013) Sexual Harassment situation in National Workplaces: A Canadian Context, Penguin Publishers, New York.

[23] EEOC (2013) Sexual Harassment situation in National Workplaces: A Chilean Context, Penguin Publishers, New York.

[24] EEOC (2013) Sexual Harassment situation in National Workplaces: A United Kingdom Context, Penguin Publishers, New York.

[25] EEOC (2013) Sexual Harassment Situation in National Workplaces: An Indian Context, Penguin Publishers, New York.

[26] EEOC (2013) Sexual Harassment situation in National Workplaces: A Kenyan Context, Penguin Publishers, New York.

[27] EEOC (2013) Sexual Harassment situation in National Workplaces: A South African Context, Penguin Publishers, New York.

[28] EEOC (2013) Sexual Harassment situation in National Workplaces: A Zambian Context, Penguin Publishers, New York. 
Perceived Risk of Sexual Harassment and Protective Behaviours among Zambian Soldiers in Selected Military Camps

[29] Elizabeth, S. and Brian, K. H. (2003). "How to Prevent Sexual Harassment in the Work Place." Equal Opportunities International, Volume 22 ISS: 2Pp 59 - 66.

[30] European Commission (2006); Sexual Harassment in the Work Place in the European Union. Directorate - General for employment industrial Relations and social affairs unity V/DS.

[31] Equal Employment Opportunity Commission (2009).Work Place Discrimination: A Fight for Non Discrimination for Gender, Penguin Publishers, New York.

[32] Fairbank J, Hansen, F. and Fittelin, G. (1991). Sexual Harassment Nature and its Effects. Sage Publishers London.

[33] Ferrer R, Klein W and Sheran P. (2016) Tripartite Model of risk perceptions (Tririsk): Distinguishing deliberative, affective and experiential components of Perceived Risk, Annals of behavior medicine, Volume 50, Issue (5), pages 563-663

[34] Fitzgerald L F, Drasgow F and Magley V J (2009) Sexual harassment in the armed forces: A test of an integrated model, Military Psychology Journal, Volume 11, Issue (3) pages 329-343.

[35] Fitzgerald L F, Drasgow F and Gelfard M J (1995) The structure ofSexual harassment : A confirmatory analysis, Military Journal of vocational behavior, Volume 47, Issue (2) pages 164-179.

[36] Foucault, M. (1975).Gender Power Relations Theories, University of Chicago Press, Chicago.

[37] Gallagher.G. (2008).Sexual Harassment and the US. Army: A Survey of Selected Military Academies, University of Kansa Publications, Kansas.

[38] Germims, S. and Pilcher, J. (2014). Fifty Key Concepts in Gender, Sage, London.

[39] Gil, B. (2003) Sexual Harassment of Nurses and Nursing Students, Journal of Advanced Nursing 42(6): 637-44.

[40] Gilman, G. (2005). Nature of Sexual Harassment and Assault in Work Places.Sage Publications, New York.

[41] Giorgi, A et al. (1985), Phenomenology and Psychological ResearchDuquesne University Press, Pittsburg.

[42] Glasser B and Strauss A. (1967) The discovery of Grounded Theory: Strategies for Qualitative Research, Aldine Transaction Publishers, and London.

[43] Graves R (2000) Qualitative Risk Assessment and Analysis, PM Network, Volume 14 Issue (10) Pages 6166.

[44] Guba, I. and Lincolns (1994) Qualitative Research, Sage Publications, London.

[45] GRZ (2005) Sexual Harassment SI no.15 section 137a, Government Printers, Lusaka.

[46] GRZ (2015) Gender Equity and Equality SI no.22, section 39/40 sexual harassment, Government Printers, Lusaka.

[47] GRZ (2017) Seventh National Development Plan (SNDP 2017-2021), Government Printers, Lusaka.

[48] Hart, C. (2013). Doing a Literature Review: Release the Social Science imagination, Sage Publications, London.

[49] Harris R.J., McDonald D.P and Sparks C.S (2017) Sexual Harassment in the military :individual experiences, demographics and organizational contexts, Journal of Armed Forces and Society, Doi:10.1177/0095327x16687069, pages 1-19

[50] Holloway, I. and Wheeler, S. (1996). Qualitative Research for Nurses, Hartinolis Ltd, London.

[51] Hoover, G (2000) “Global Military Recruitments and Sexual Harassment in Military academies. http://www.wells.edu.HR.htm.

[52] Hughes, J. O. and Sandler, B.R. (2002).In Case of Sexual Harassment: A Guide for Women Students, Centre for Women Policy Studies Unpublished.

[53] Homans, George C. (1962). 'Social Behaviour and sexual harassment: It's Elementary, Harcourt, Brace and World Inc., New York.

[54] Isaac, D. P (2002). Perceptions of sexual harassment amongst university students: A case study of the South African Military Academy, University of Stellenbosch (thesis), Cape town.

[55] Janson, H. (2013) Accountability for Crimes of Sexual Harassment and Abuse committed by South African Peace Keeping Troops, Witwatersrand University, Johannesburg.

[56] Jana, P. L. (2003). Why Women Don't Report Sexual Harassment: A Case Study of The Elite Military Institution, Gender Issues Journal Volume 21, Issue 3, Pages 3-30.

[57] Kamal, D. Omotosho. F. and Elizabeth, S. (2001). Coping Strategies of Sexual Harassment, Heresies Publishers, New Delhi. 
[58] Kamir, D. (1998). “Israel's 1998 Sexual Harassment Law; Prohibiting Sexual Harassment, Sexual Stalking and degradation based on sexual orientation in the work place and in all social settings", International Journal of Discrimination and Law, 2005, Vol. 7 Pp 515-336.

[59] Kibeomam, H (2006). Sexual Harassment and its Nature (42) 305 - 310.

[60] Kim, K.T; Lee, H.C; Lee, S.G; Han, K.T and Park, E. C. (2016).The Influence Of Sexual Harassment On Mental Health Among Female Military Personnel Of The Republic Of Korea Armed Forces, Journal of Royal Medical Corps volume 163 issue 2.

[61] Kombo, D. K. and Tromp, L. A. (2006).Proposal and Thesis Writing: An Introduction, Pauline Publication Africa, Nairobi.

[62] Krueger, R. A. (1988). Focus Groups and Interviews: A practical Guide for Applied Research Methods. Sage Publishers, London.

[63] Lazarus, R. S and Folkman, S. (1980). An Analysis of Coping of sexual harassment in a Middle Aged Community Sample. Journal of Health and Social Behaviour, 21, pp. 219 - 239 Abstract - MEDLINE.

[64] Leardman C.A, Pietrucha A, Magrudah K .M , Gackstenter G and Smith T C (2013) Combat deployment is associated with sexual harassment or sexual assault in a large female military cohort, Women's Health Issues Journal, Volume 23 Issue (4) pages e215-e223

[65] Leedy, A. and Omorod, J. F. (2005).Practical Research Planning and Design.Merrill Prentice Publishers, New Jersey.

[66] Leskinen, E. A, Cortina L.M and Kabat, D. B (2011). Gender Harassment: Broadening our Understanding Of Sex Based at Work (Legal and Military). Law and Human Behaviour Journal, Volume 35 Issue 1 Pages 25-39.

[67] Maree K and Westhuizen V (2009) Head start in designing research proposals in the social sciences, Juta and company limited, Cape Town.

[68] Matakala, J. M. (2015). Sexual Harassment and Experiences of Female Soldiers on the UN Peace Keeping Operations, Dissertation, University of Zambia, Lusaka.

[69] Matakala, J.M, Akabondo, I.N, Macwan'gi, M, Ndubani, P, and Phiri, A.N (2018).Gendered Phenomenological perceptions on the characteristics of Sexual Harassment among Soldiers in Eastern Zambia. International Journal of Humanities Social Sciences and Education Volume 5 issue 7 page 91101.

[70] Matakala, J.M, Macwan'gi, M, Ndubani, P, and Phiri-Namakando A (2018).Developing a Gendered Participatory Preventive Approach to prevent of Sexual Harassment among Soldiers in Zambia. International Journal of Humanities Social Sciences and Education Volume 5 issue 1 page 1- 8.

[71] Mary, L. (2006). Sexual Harassment: Principle of Equal Treatment for men Women in Vocational Training, University Education, Colleges and Hospital Training, Sage Publications, New York.

[72] Mary, R. (2005) Dealing with Sexual Harassment and Coping with it: A Systems ApproachPsychological Journal (36) 27-38.

[73] Merriam, C. (2012) Developing a female preventive participatory approach to prevent sexual harassment on female officer cadets in Canada, Journal of Social issues, 2012. 62 (2) 212-235.

[74] Miller L L. (1997) Not just weapons of the weak: Gender harassment as a form of protest for army men, Social Psychology Quarterly Journal, Volume 60, Issue (1), Pages 32-51.

[75] Mirriam, C. 2015 Developing a female prevention model for sexual harassment a case of female cadets in the Canadian Defense forces, Journal of issues, 2015, 72 (2) pp 52-89.

[76] Mills, A. J. (1992) Dealing with Sexual Harassment Behavior and Analysis, Journal of Sexual Harassment Analysis, (56), 103-308

[77] Msabila, T. and Nalaila, G. (2013).Research Proposal and Dissertation Writing: Principles and Practice, Nyambari Publishers, Dar es Salaam.

[78] Minichioelo, V. Aroni, R, Time well, E. and Alexander, L. (1990).Qualitative data analysis in depth Interviewing: Qualitative Approach, Sage Publishers, Thousand Oaks.

[79] Moody, L E. (1990) Advancing nursing science through research, Sage publications, California.

[80] Mouton J (2001) How to succeed in your master's and doctoral studies: A south African resource book, Van Schaik publishers, cape town.

[81] Morrow, P. C. Me-Elroy, J. C. and Philips, C.M (2004).Sexual Harassment Behaviours and Work Related Perceptions and Attitudes, Journal of Vocational Behaviour, (45), 295-309.

[82] Moser, C. O. N. (1993). Gender Planning and Development: Theory, Practice and Training. Routledge Publishers, London. 
Perceived Risk of Sexual Harassment and Protective Behaviours among Zambian Soldiers in Selected Military Camps

[83] Murdock, M. (1998).Measuring sexual harassment in the US Army: Development and validation of sexual harassment inventory. Journal of violence and victims' volume 13, issue 3, p 203-216.

[84] Nikolov M. V (2017) United States Army drill sergeants response to sexual harassment and assault of Gay, Lesbians and Bisexual recruits, Walden University (thesis) scholar works (Dissertations and Doctoral studies).

[85] O’donohue, W. (2007).Sexual Harassment: Theory Research and Treatment. Allyn and Bacon, Boston.

[86] O'Hare E A and O'donohue, W. (1998) Sexual Harassment: Identifying risk factors, Archives of sexual behavior, Volume 27, Issue (16), pages 561-580.

[87] Pallavi, P. (2014) The Sexual Harassment of Women at Workplace (Prevention, Prohibition and Redressal) Act 2013 (India), Press Bureau Publishing, Calcutta, India.

[88] Parahoo, K. (1996). Nursing Research, Principles, Process and Issues, Macmillan Press Ltd London.

[89] Patton, M.Q. (2002). Qualitative Research and Evaluation Methods. Thousand Oaks, London.

[90] Pershing J L (2003) Why women don't report sexual harassment : A case of an elite military institution, Gender Issues Journal ,Volume 21, Issue(3), Pages 3-20

[91] Penal Code Act (2013) - National Assembly of Zambia, Sexual Harassment, Laws of Zambia, 29 October, 2013.

[92] Punch, K. F. (2012). Introduction to Social Research: Quantitative and Qualitative Approaches, Sage Publications, London.

[93] Pryor, J. B (2010).The Psycho Social Impact of Sexual Harassment On Women in The US Military, Basic and Applied Social Psychology 17(4):581-603.

[94] Pryor, J. (1997). Sexual Harassment Proclivities in Men, Sex Roles, (17) 269 - 290.

[95] Pryor, J.B. and Whalen, N. J. (2007).A Typology of Sexual Harassment. Characteristics of Harassers and the Social Circumstances under which Harassers Occur. Journal Psychology, (50) 308 - 326.

[96] Rosen L N and Martin L (1998).Psychological Effects of Sexual Harassment and Organizational Climate Among United States Army Soldiers Military Medicine Journal Volume (13) Issue 3 Pages 209-213.

[97] Rosen L N and Martin L (1998).Childhood Maltreatment History as a Risk Factor of Sexual Harassment Among United States Army Soldiers Military Medicine Journal Volume (13) Issue 3 Pages 269-286.

[98] Rosen L N and Martin L (2000).Personality Characteristics that increase vulnerability to sexual harassment among US army soldiers, Military Medicine Journal Volume (165) Issue 10 Pages 709-713.

[99] Spak, M. I. and McCarty, A.M. (2004) Effects of the military culture on responding to sexual harassment: The warrior mystique, Nebraska Law Review Journal Volume 83 issue 1 pages

[100]Settles, J. H., Yang, I. S. and Yap, S. C. (2014) Sexual Differences in Outcomes and Sexual Harasser Characteristics associated with frightening Sexual Harassment among Service Personnel, Occupational Health Psychology Journal, 2014, Volume (19) pg. 133-142

[101]SCGR, (2008).Measures of Stopping Sexual Harassments and Assaults in Peace Keeping Operations, United Nations Publication, New York.

[102] Schuyler A S, Klemmer C, Mamey M R, Shrager S M, Goldbach J T, Holloway I W and Castrol C A (20 ) Experiences of sexual harassment, stalking, and sexual assault during military service among LGBT and Non-LGBT service members, Journal of Traumatic Stress, Volume 33, Issue (3), Pages 20-63.

[103]Smith J A, Flowers P and Larkin M (2009) Interpretive Phenomenology Analysis, Sage publications, London.

[104]Smith J A, and Osborne M (2014) Interpretive Phenomenology Analysis and Health Psychology ,Routledge publications, London.

[105]Tae, K.K, Lee, S. G, Han, K and Park, E (2012) The influence of sexual harassment on mental health among female military personnel of the republic of Korea Armed Forces, Journal of Royal Medical Corps Volume (163) issue 2 pages 55-72.

[106]Tangri , S.A, and Johnson, L.B (2002) Three explanatory models for sexual harassment in the US Military Journal of Social Issues Volume 38) issue (4) Pages 33-54.

[107]Turchik J and Susan, W (2010) Sexual Harassment, Assault in the United States Military: A review of literature and recommendations for the future, Aggression and Violent Behaviour Journal Volume (15) Pages 266-277.

[108]UNSC, (2008).Challenges for the Deployment of United Nations Peace Keepers in Mission Areas, United Nations, New York.UNSC, (2008).

[109]UNFPA (2018) United Nations Funded Population Agency :Zambia annual report, Government Printers, Lusaka. 
[110]Valerie, S.A and Cynthia, T.J (2016) Sexual harassment and Assault in the US Military: A Review of Policy and trends, Military Medicine Journal Volume (181) Issue 1 Pages 20-29.

[111]Wijk, V.C, Finchelesk, K.G, and Tredoux, C (2009) Sexual Harassment of women in the South African Navy, South African Journal Volume (60) Pages 53-60.

[112]World Health Oranisation (2014) Sexual and Reproductive Health, United Nations, New York.

[113]Zandonda. P. N. N. (2010). A Study Sexual Harassment of Women in Work Places: A Study of Selected Public and Private Organizations in Ndola Zambia, University of Zambia Thesis, Lusaka.

\section{APPENDICES}

\section{Appendix 1: Self/Researcher administered \{Sexual Harassment Experience Questionnaire [SHEQ]\}.}

\section{Topic: Perceived Risk of Sexual Harassment And Protective Behaviour Among Zambian Soldiers In Selected Military Camps.}

Dear Potential Participants,

This serves to inform you that I am a Doctor of Philosophy student at the University of Zambia under Gender Studies. The topic I am studying Perceived Risks Of Sexual Harassment And Protective Behaviour Among Zambian Soldiers In Selected Military Camps.

Therefore, this questionnaire is meant to come up with responses regarding your understanding of the concepts of perceived risk and sexual harassment. It is also aimed at establishing whether you protect or not protect yourself from risk of being sexually harassed by providing answers from the under listed questions.

Instructions: Kindly answer all questions by way of ticking the correct one.

1. Do you agree that the concept of perceived risk can either mean (low, medium or high) awareness towards any threat (such as being sexually harassed)?
(a)Yes
(b) No
(c) Not aware

2. Are you aware that Sexual Harassment includes unwelcome verbal, nonverbal and physical actions?
(a)Yes
(b) No
(c) Not aware

3. Do you protect yourself against the risk of being sexually harassed?
(a)Yes
(b) No
(c) Not aware

4. What is your sex?
(a) Male
(b) Female

5. Circle any of the seven non-commissioned rank categories that define a soldier were you belong. 1.Privates,

2. Lance Corporal,

3. Corporal,

4. Sergeant,

5. Staff Sergeant,

6. Warrant Officer Class Two

7. Warrant Officer Class One.

For any clarification or queries my cell is +260 974637403 my email address is johnmatakala2017@yahoo.com.

Thanking you in advance kindly send a text when you are through to the contact above.

\section{Appendix 2: Informed Consent Form}


Topic: Perceived Risks of Sexual Harassment And Protective Behaviour Among Zambian Soldiers In Selected Military Camps.

Dear participants,

This serves to give you an understanding of the research and procedures that will be followed.

Similar information in this form will be read to you alongside the questions with regard to each objective and its research instrument.

Further the implications for your participation are explained below, finally you are asked to sign this form to indicate that you have agreed to participate in this exercise.

Thanking you in advance.

\section{Description}

This is an educational research. The researcher is a student at the University of Zambia pursuing a Doctor of Philosophy Degree in Gender Studies.

This research is a major requirement for the researcher to complete this program. Therefore, this study is purely academic.

\section{Purpose}

The researcher's topic is: Perceived Risks Of Sexual Harassment And Protective Behaviour Among Zambian Soldier In Selected Military Camps.

The researcher is interested in understanding the how both the harassed (victims) and non-harassed (non-victims) Perceived Risks of sexual harassment and the resultant protective behaviours. The evidence could be used to prevent or reduce sexual harassment among Zambian Soldiers in selected military camps. He therefore is interested in learning how participants perceive the risk of sexual harassment from both the harassed (victims) and non-harassed (non-victims) and there protective behaviours.

\section{Consent}

Participation in the exercise is voluntary. You are free to decline to participate in this exercise.

\section{Confidentiality/Sharing of Findings}

All data collected from this research is treated with utmost confidentiality. Participants are assured that they will remain anonymous and untraceable in this research.

It is against this background that participants will only be identified through a number and not by the actual name but a number or pseudo names will be used.

\section{Rights of Participants}

All efforts will be taken to ensure that the rights of participants as per research ethics are protected and respected. Participants are assured that they are free to ask for clarification at any point of the exercise and to inform the researcher if they feel uncomfortable about any procedure in the research.

Your consent to this request will be highly valued and appreciated.

\section{Uses of Information}

The information that will be got from you will be got from you may help in decision making for the military authorities aimed at improving the situation of the topic under study. Most of all it may also equip military individuals with formal skills for sexual harassment individual protective behaviors.

\section{Individual Risks}

There are no risks because as per research ethics anonymity and confidentiality will be assured both during and after the research has been conducted. Participants will therefore remain untraceable..

\section{Benefits to Participants}

There will be no direct benefits but your participation is likely to help us in understanding how both the harassed (victims) and non-harassed (non-victims) Perceived Risks of sexual harassment and the resultant protective behaviours. 


\section{Reimbursements}

The researcher will not provide any incentive to take part in the research. However, we will give you [provide a figure if money is involved] for time and travel expenses if and when applicable.

\section{Duration/ Research Intervention}

This research will take up to three months in total. During this time we shall visit you up to three or four times with each of the face to face oral interviews taking up to one and half hours each. This therefore will involve your physical participation both at individual or group level. You have been purposefully selected to help in understanding of the topic under discussion.

John Mwitumwa Matakala -PhD Research Fellow.

Computer Number 2016144605

\section{Declaration of Consent}

I have read or head and fully understand this document concerning the research and its procedures. Therefore, voluntarily I have agreed to participate in this study.

Participant number .......Signature ................ Date.

\section{Appendix 3: Semi-Structured Interview Guide For Soldiers That Include Both Male And Female Victims/Non-Victims Of Sexual Harassment.}

Topic: Perceived Risks of Sexual Harassment and Protective Behaviour Among Zambian Soldiers In Selected Military Camps.

Participation Criteria: Only those who provided information from the \{Sexual Harassment Experience Questionnaire\} are eligible

Dear participant,

My names are John Mwitumwa Matakala and I am a University of Zambia student pursuing a Doctor of Philosophy in Gender Studies. As a fulfillment of the requirements for the program, students are required to research on topics of their choice;Perceived Risks of Sexual Harassment and Protective Behaviour among Zambian Soldiers in Selected Military Camps.

The topiccaptured my interest being a gender student as well as a soldier.

You have been purposefully selected to this individual face to face formal conversation for the study inorder to understand the topic above. The researcher fills your affirmative response from theSexual Harassment Experience Questionnaire [SHEQ] towards the concepts of perceived risk and sexual harassment will give to an in-depth understanding in relation to your protective behavioral situation as a female or male soldier, in the Non-commissioned ranks.

In addition, information collected through this study is strictly for academic purposes only and therefore shall be kept confidential and no name or any identity shall be attributed to you. Furthermore, you are free to choose to participate in this research and you can also choose to pull out any time you feel uncomfortable.

I would appreciate if you could spare sometime to answer some questions in my interview guide because your participation is highly valued.

During the interview further probing or follow up questions based on your answers will also be asked.

\section{Question One:}

(1) How is the situation of perceived risk of being sexually harassed among Zambian soldiers in selected military camps?

\section{Question Items (for question1)}

- Please tell me about yourself.

- Kindly tell me your actual sexual harassment experience? 
- In your own opinion as a (victim) or (non-victim) explain what makes you Perceive (High, Medium or Low) Risk towards sexual harassment ..?

Thank you very much for your co-operation and time stay blessed I hope to hear from you again Question Two:

(2) What risk factors are associated with sexual harassment among Zambian soldiers in selected military camps?

\section{Question Items (for question2)}

- How do you think sexist/perpetrator/sexual harassers contribute to the potential risks that could increase sexual harassment among the interaction of soldiers?

- What do you think are the major individual victim potential risk factors that are associated with the occurrences of sexual harassment?

- In your own opinion describe the military potential risk factors that you think are associated with the occurrences of sexual harassment.

Thank you very much for your co-operation and time stay blessed I hope to hear from you again

\section{Question Three:}

(3) What protective behaviors do Zambian soldiers adopt against the risk factors of being sexually harassed in selected military camps?

\section{Question Items (for question 3)}

- Do you protect yourself against (low, medium or high) perceived or awareness levels of risk factors towards the possibility of being sexually harassed?

- In your own opinion why do you adopt or not adopt protective behavior against the (low, medium or high) awareness level towards the possibility of being sexually harassed in relation to (low, medium or high) severity or unpleasantness?

- What type of protective behaviors do you use against risk of sexual harassment?

Thank you very much for your co-operation and time stay blessed I hope to hear from you again

\section{Research Question Number Four (04)}

4) How do perceived risks of being sexually harassed motivate protective behaviors among Zambian soldiers in selected military camps?

\section{Thematic Sub Questions:}

- In your own opinion how does (non-victim high perceived risk) of sexual harassment motivate protective behaviors?

- How effective are the adopted protective behaviors do you use against risk of sexual harassment?

\section{AUTHOR'S BIOGRAPHY}

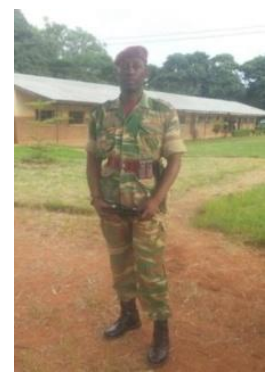

John Mwitumwa Matakala, is a Doctor of Philosophy Reasearch fellow In Gender Studies. At the University of Zambia. He is the Principal Researcher. He is Also Special Force Soldier in the Commando Unit. He is the Prinicipal Researcher.

Citation: John Mwitumwa Matakala, et.al. "Perceived Risk of Sexual Harassment and Protective Behaviours among Zambian Soldiers in Selected Military Camps" International Journal of Humanities Social Sciences and Education (IJHSSE), vol 8, no. 4, 2021, pp. 72-123. doi: https://doi.org/10.20431/2349-0381.0804009.

Copyright: (C) 2021 Authors. This is an open-access article distributed under the terms of the Creative Commons Attribution License, which permits unrestricted use, distribution, and reproduction in any medium, provided the original author and source are credited. 